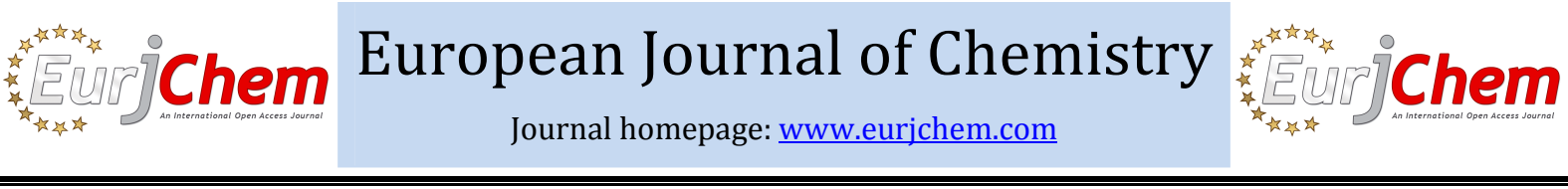

\section{Synthesis, reactions, antioxidant and anticancer evaluation of some novel coumarin derivatives using ethyl 2-(2-oxo-4-phenyl-2H-chromen-7-yloxy) acetate as a starting material}

\author{
Ahmed Hamdy Halawa a, Ahmed Abd El-Hameed Hassan a,b,*, Mohamed Ahmed El-Nassag a, \\ Mahmoud Mohamed Abd El-All a,c, GehadAbd El-Raheem Abd El-Jaleel d, \\ Essam Mohamed Eliwa a and Ahmed Hammam Bedair a
}

a Chemistry Department, Faculty of Science, Al-Azhar University, 11884, Nasr City, Cairo, Egypt

b Chemistry Department, Faculty of Medicine, Jazan Universit y, 82621, Jazan, Saudi Arabia

c Chemistry Department, Faculty of Science and Art, Al-Baha University, 1988, Al-Baha, Saudi Arabia

d Pharmacology Department, National Research Center, 12622, Dokki, Cairo, Egypt

*Corresponding author at: Chemistry Department, Faculty of Science, Al-Azhar University, 11884, Nasr City, Cairo, Egypt. Tel.: +2.0100.4353504. Fax: +2.022.2926356. E-mail address: ahmed hassan7979@hotmail.com (A.A.E. Hassan).

\section{ARTICLE INFORMATION}

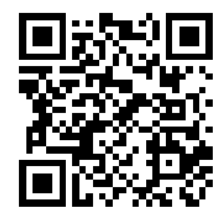

DOI: $10.5155 /$ eurjchem.5.1.111-121.860

Received: 15 June 2013

Received in revised form: 15 September 2013

Accepted: 15 September 2013

Online: 31 March 2014

\section{KEYWORDS}

\section{Triazole}

Oxadiazole

Cytotoxicity

Benzoimidazole

Antioxidant activity

Coumarinacetohydrazide

\section{Introduction}

The coumarin nucleus is frequently found in bioactive compounds and plays an important role in biochemical processes. The cytotoxic activities of the coumarin derivatives were tested in several human tumor cell lines [1-7]. In addition, the antimicrobial, antitumor, anti-inflammatory, antimalarial and anti-HIV activities of hydrazide compounds on tumor cell lines have been observed and recently reported [8-14], Moreover, some synthetic oxadiazole derivatives exhibit a range of pharmacological activities [15-18]. The biological and medicinal activities of triazole moieties have stimulated considerable interest in the synthesis of derivatives of this ring system [19-25]. In view of the above observations and in continuation of our previous works in heterocyclic chemistry, we report herein the synthesis of some new derivatives of these ring systems incorporated with 4-phenylcoumarin moiety, using ethyl 2-(2-oxo-4-phenyl-2H-chromen-7-yloxy) acetate (1) [26] as a key starting material. A selected series of these compounds were investigated for their antitumor activities.

\section{Experimental}

\subsection{Instrumentation}

All melting points, antioxidant and anticancer activities are uncorrected. IR spectra (KBr) were recorded on FT-IR 5300 spectrometer and Perkin Elmer spectrum RXIFT-IR system ( $v$, $\mathrm{cm}^{-1}$ ). The ${ }^{1} \mathrm{H}$ NMR spectra were recorded in (DMSO-d6) at 300 $\mathrm{MHz}$ on a Varian Mercury VX-300 NMR spectrometer $(\delta, \mathrm{ppm})$ using TMS as an internal standard. ${ }^{13} \mathrm{C}$ NMR spectra were recorded on Varian Mercury VX 300 NMR using DMSO- $d_{6}$ as solvent and TMS as an internal standard. Mass spectrum was obtained on GC MS-QP 1000 EX mass spectrometer at $70 \mathrm{eV}$. Elemental analyses were carried out by the Microanalytical Research Center, Faculty of Science, Cairo University and AlAzhar University. 


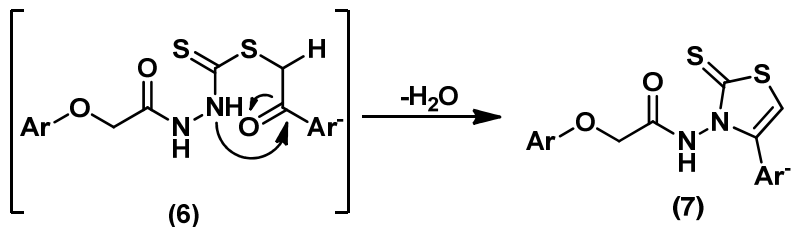

(6)

(7)<smiles>O=C(Br)CBr</smiles><smiles>CC(C)NCC[Te]OCc1n[nH]c(=S)n1N</smiles><smiles>CCOC(=O)CO[Bi]</smiles>

(1)<smiles>Cc1ccc2c(-c3ccccc3)cc(=O)oc2c1</smiles><smiles>Cc1ccc([N+]([O])=[Zn])cc1</smiles>

(3)<smiles>C=C1NN=C(C(=C)C)O1</smiles>

(4)
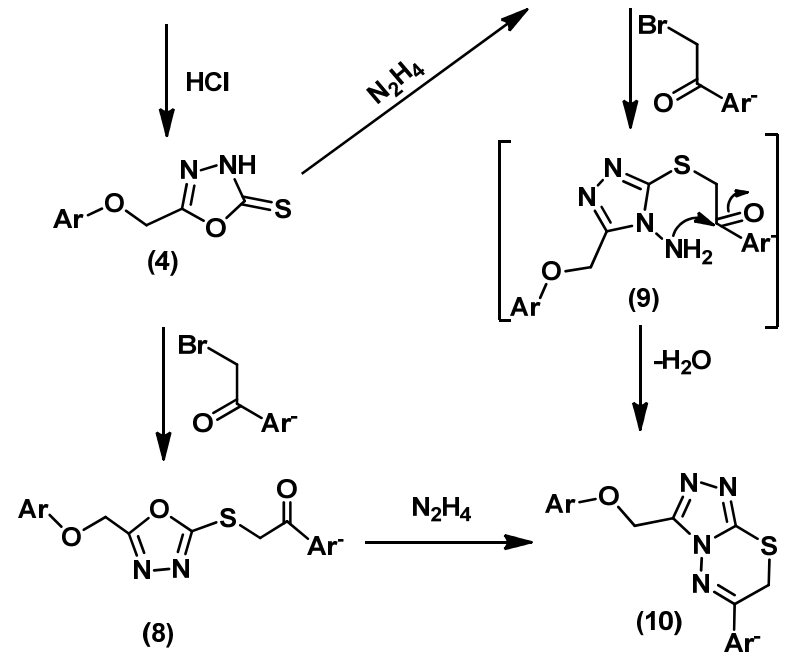

Scheme 1

\subsection{Synthesis}

\subsubsection{Synthesis of 2-(2-oxo-4-phenyl-2H-chromen-7-yloxy) acetohydrazide (2)}

A mixture of compound $\mathbf{1}(0.01 \mathrm{~mol})$ and hydrazine hydrate $(0.02 \mathrm{~mol})$ in ethanol $(30 \mathrm{~mL})$ was refluxed for $8 \mathrm{~h}$. The reaction mixture was concentrated and left to cool (Scheme 1). Color: Colorless. Yield: $80 \%$. M.p.: $188-190{ }^{\circ} \mathrm{C}$. FT-IR (KBr, v, $\left.\mathrm{cm}^{-1}\right)$ : 3340, $3254\left(\mathrm{NH}_{2}, \mathrm{NH}\right), 1716(\mathrm{C}=0$, lactone), $1690(\mathrm{C}=0$, amide). ${ }^{1} \mathrm{H}$ NMR (300 MHz, DMSO- $\left.d_{6}, \delta, \mathrm{ppm}\right): 9.43(\mathrm{~s}, 1 \mathrm{H}, \mathrm{NH}$ exchangeable with $\left.\mathrm{D}_{2} \mathrm{O}\right), 6.96-7.58(\mathrm{~m}, 8 \mathrm{H}, \mathrm{Ar}-\mathrm{H}), 6.25(\mathrm{~s}, 1 \mathrm{H}, \mathrm{H}-$ 3), $4.63\left(\mathrm{~s}, 2 \mathrm{H}, \mathrm{OCH}_{2}\right), 4.36\left(\mathrm{~s}, 2 \mathrm{H}, \mathrm{NH}_{2}\right.$ exchangeable with $\left.\mathrm{D}_{2} \mathrm{O}\right)$. MS (EI, $m / z(\%)$ ): $310\left(\mathrm{M}^{+}, 77\right)$. Anal. calcd. for $\mathrm{C}_{17} \mathrm{H}_{14} \mathrm{~N}_{2} \mathrm{O}_{4}$ : C, 65.80; H, 4.55; N, 9.03. Found: C, 65.74; H, 4.43; N, 8.91\%.

\subsubsection{Synthesis of potassium 2-[2-(2-oxo-4-phenyl-2H- chromen-7-yloxy) acetyl]hydrazinecarbodithioate (3)}

To a solution of 2-(2-oxo-4-phenyl-2H-chromen-7-yloxy)acetohydrazide $(2,0.01 \mathrm{~mol})$ in ethanol $(30 \mathrm{~mL})$, a solution of potassium hydroxide $(0.01 \mathrm{~mol})$ in water $(5 \mathrm{~mL})$ and carbon disulfide $(0.01 \mathrm{~mol})$ were added. The reaction mixture was heated under reflux for $3 \mathrm{~h}$, the resulting solid on heating was collected by filtration, washed with ether and dried to afford $2.8 \mathrm{~g}$ of the potassium salt 3 (Scheme 1). Color: yellow. Yield: $90 \%$. M.p.: $>360^{\circ} \mathrm{C}$. FT-IR $\left(\mathrm{KBr}, v, \mathrm{~cm}^{-1}\right): 3320,3266(\mathrm{NH}), 1698$ $(\mathrm{C}=0) .{ }^{1} \mathrm{H}$ NMR $\left(300 \mathrm{MHz}\right.$, DMSO- $\left.d_{6}, \delta, \mathrm{ppm}\right): 6.57-7.58(\mathrm{~m}, 10 \mathrm{H}$, Ar-H and 2NH), 6.25 (s, 1H, H-3), 5.19 (s, 2H, $\mathrm{OCH}_{2}$ ). MS (EI, $m / z(\%)): 424\left(\mathrm{M}^{+}, 61\right)$. Anal. calcd. for $\mathrm{C}_{18} \mathrm{H}_{13} \mathrm{KN}_{2} \mathrm{O}_{4} \mathrm{~S}_{2}$ : C, 50.92; H, 3.09; N, 6.60. Found: C, 50.85; H, 3.00; N, 6.51\%.

\subsubsection{Synthesis of 7-[(5-thioxo-1,3,4-oxadiazol-2-} yl)methoxy]-4-phenyl-2H-chromen-2-one (4)

The potassium salt $3(0.01 \mathrm{~mol})$ was dissolved in aqueous potassium hydroxide solution $(0.01 \mathrm{~mol})$ in water $(10 \mathrm{~mL})$ and refluxed for $2 \mathrm{~h}$ then cooled. The resulting reaction mixture was treated with dilute hydrochloric acid till $\mathrm{pH}=\sim 4$. The resulting solid was collected by filtration, washed with water, and recrystallized from ethanol to give compound 4 (Scheme 1). Color: yellow. Yield: $76 \%$. M.p.: $185-187^{\circ} \mathrm{C}$. FT-IR $\left(\mathrm{KBr}, v, \mathrm{~cm}^{-1}\right)$ : $1686(\mathrm{C}=0) .{ }^{1} \mathrm{H}$ NMR $\left(300 \mathrm{MHz}\right.$, DMSO- $\left.d_{6}, \delta, \mathrm{ppm}\right): 14.23(\mathrm{~s}, 1 \mathrm{H}$, $\mathrm{NH}), 7.02-7.58(\mathrm{~m}, 8 \mathrm{H}, \mathrm{Ar}-\mathrm{H}), 6.28(\mathrm{~s}, 1 \mathrm{H}, \mathrm{H}-3), 5.42(\mathrm{~s}, 2 \mathrm{H}$ $\mathrm{OCH}_{2}$ ). MS (EI, $\left.m / z(\%)\right): 352\left(\mathrm{M}^{+}, 51\right)$. Anal. calcd. for $\mathrm{C}_{18} \mathrm{H}_{12} \mathrm{~N}_{2} \mathrm{O}_{4} \mathrm{~S}: \mathrm{C}, 61.35 ; \mathrm{H}, 3.43 ; \mathrm{N}, 7.95$. Found: C, 61.28; H, 3.35; N, $7.87 \%$.

2.2.4. Synthesis of 7-[(4-amino-5-thioxo-4, 5-dihydro-1H-1, 2 , 4-triazol-3-yl) meth-oxy]-4-phenyl-2H-chromen-2-one (5)

Method A: To a solution of potassium salt $3(0.01 \mathrm{~mol})$ in ethanol $(20 \mathrm{~mL})$ and water $(10 \mathrm{~mL})$, hydrazine hydrate $(0.2$ $\mathrm{mL}$ ) was added. The reaction mixture was heated under reflux for $2 \mathrm{~h}$, then left to cool, then poured into crushed ice. The resulted reaction mixture was acidified with dilute hydrochloric acid. 


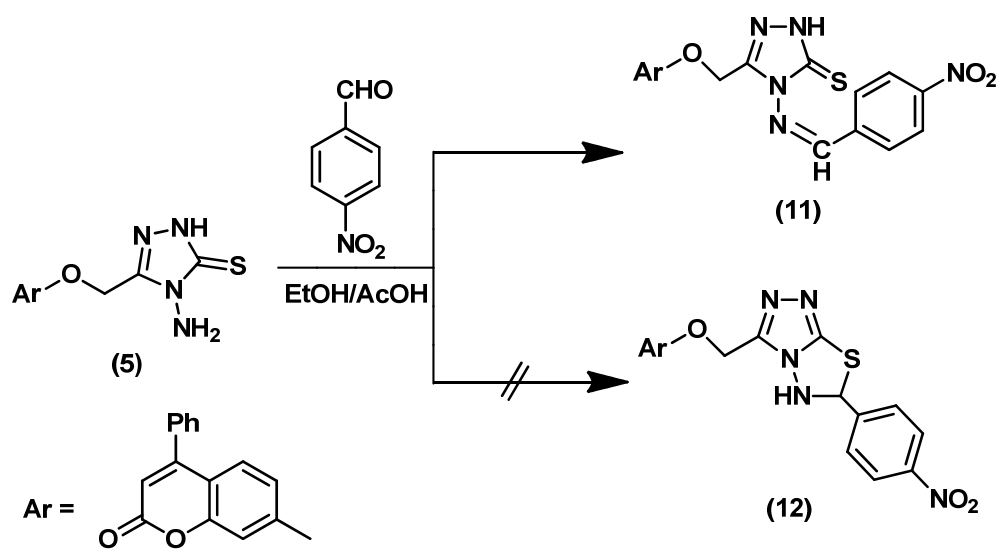

Scheme 2

The resulting solid was collected by filtration, washed with water and recrystallized from dioxane (Scheme 1). Color: White. Yield: $77 \%$. M.p.: $222-224^{\circ} \mathrm{C}$. FT-IR (KBr, v, $\mathrm{cm}^{-1}$ ): 1686 $(\mathrm{C}=0), 3384,3298\left(\mathrm{NH}_{2}\right), 3182(\mathrm{NH}), 1698(\mathrm{C}=0) .{ }^{1} \mathrm{H}$ NMR $(300$ MHz, DMSO- $\left.d_{6}, \delta, p p m\right): 13.84(\mathrm{~s}, 1 \mathrm{H}, \mathrm{NH}$ exchangeable with $\left.\mathrm{D}_{2} \mathrm{O}\right), 6.99-7.56(\mathrm{~m}, 8 \mathrm{H}, \mathrm{Ar}-\mathrm{H}), 6.25(\mathrm{~s}, 1 \mathrm{H}, \mathrm{H}-3), 5.67$ (b, $2 \mathrm{H}, \mathrm{NH}_{2}$ exchangeable with $\left.\mathrm{D}_{2} \mathrm{O}\right), 5.27\left(\mathrm{~s}, 2 \mathrm{H}, \mathrm{OCH}_{2}\right) .{ }^{13} \mathrm{C} \mathrm{NMR}(300 \mathrm{MHz}$, DMSO-d $\left._{6}, \delta, \mathrm{ppm}\right): 166.79,160.61,159.79,155.17,154.93$, $147.49,134.83,129.61,128.79,128.35,127.85,112.80,112.48$, 111.78, 102.35 and 59.73. MS (EI, $m / z(\%)): 366\left(\mathrm{M}^{+}, 25\right)$. Anal. calcd. for $\mathrm{C}_{18} \mathrm{H}_{14} \mathrm{~N}_{4} \mathrm{O}_{3} \mathrm{~S}$ : C, 59.01; H, 3.85; N, 15.29. Found: C, 58.89; H, 3.78; N, $15.18 \%$.

Method B: A mixture of 7-[(5-thioxo-1,3,4-oxadiazol-2yl)methoxy]-4-phenyl-2H-chromen-2-one (4) (0.01 mol) in ethanol $(20 \mathrm{~mL})$ and hydrazine hydrate $(0.2 \mathrm{~mL})$ was heated under reflux for $2 \mathrm{~h}$, then left to cool and poured onto crushed ice. The reaction mixture was acidified with dilute hydrochloric acid till $\mathrm{pH}=\sim 4$. The resulting solid was collected by filtration, washed with water and recrystallized from dioxane, to give compound $\mathbf{5}$.

\subsubsection{Synthesis of N-(4-(4-nitrophenyl)-2-thioxothiazol- 3(2H)-yl)-2-((2-oxo-4-phenyl-2H-chromen-7-yl)oxy) acetamide (7)}

A mixture of potassium salt $3(0.01 \mathrm{~mol})$ and $p$-nitro phenacyl bromide $(0.01 \mathrm{~mol})$ in $\mathrm{N}, \mathrm{N}$-dimethylformamide (DMF) $(15 \mathrm{~mL})$ was heated under reflux for $3 \mathrm{~h}$, then left to cool and poured onto crushed ice. The precipitated solid was collected by filtration, washed with ethanol, dried and recrystallized from dioxane (Scheme 1). Color: Yellow. Yield: 54\%. M.p.: 170$172{ }^{\circ} \mathrm{C}$. FT-IR (KBr, v, cm $\left.{ }^{-1}\right): 3315(\mathrm{NH}), 1716,1700(\mathrm{C}=0) .{ }^{1} \mathrm{H}$ NMR (300 MHz, DMSO- $\left.d_{6}, \delta, \mathrm{ppm}\right): 10.62(\mathrm{~s}, 1 \mathrm{H}, \mathrm{NH}), 6.80-8.38$ (m, 13H, Ar-H and CH-thiazole), 6.29 (s, 1H, H-3), 5.18 (s, 2H, $\mathrm{OCH}_{2}$ ). Anal. calcd. for $\mathrm{C}_{26} \mathrm{H}_{17} \mathrm{~N}_{3} \mathrm{O}_{6} \mathrm{~S}_{2}$ : C, 58.75; H, 3.22; N, 7.91. Found: C, 58.70; H, 3.14; N 7.82\%.

\subsubsection{Synthesis of 7-\{[5-(2-(4-nitrophenyl)-2-oxoethylthio)-} 1,3,4-oxadiazol-2-yl]methoxy\}-4-phenyl-2H-chromen-2-one (8)

A mixture of 7-[(5-thioxo-1,3,4-oxadiazol-2-yl)methoxy]-4phenyl-2H-chromen-2-one (4) (0.01 mol), p-nitrophenacyl bromide $(0.01 \mathrm{~mol})$ and anhydrous potassium carbonate $(0.01$ $\mathrm{mol})$ in dry acetone $(15 \mathrm{~mL})$ was refluxed for $6 \mathrm{~h}$. The formed precipitate was filtered off and washed with acetone. The filtrate was distilled under reduced pressure till dryness then the residue was treated with water. The solid formed was collected by filtration, washed with water, dried and recrystallized from dioxane to give compound 8 (Scheme 1).
Color: Yellow. Yield: $64 \%$. M.p.: $110-112{ }^{\circ} \mathrm{C}$. FT-IR (KBr, v, cm 1): $1710(\mathrm{C}=0) .{ }^{1} \mathrm{H}$ NMR (300 MHz, DMSO- $\left.d_{6}, \delta, \mathrm{ppm}\right): 6.51-8.39$ (m, 12H, Ar-H), 6.14 (s, 1H, H-3), $5.21\left(\mathrm{~s}, 2 \mathrm{H}, \mathrm{OCH}_{2}\right), 4.60(\mathrm{~s}, 2 \mathrm{H}$, $\mathrm{CH}_{2}$ ). MS (EI, $\left.m / z(\%)\right): 515\left(\mathrm{M}^{+}, 25\right)$. Anal. calcd. for $\mathrm{C}_{26} \mathrm{H}_{17} \mathrm{~N}_{3} \mathrm{O}_{7} \mathrm{~S}: \mathrm{C}, 60.58 ; \mathrm{H}, 3.32 ; \mathrm{N}, 8.15$. Found: $\mathrm{C}, 60.48 ; \mathrm{H}, 3.20$; N, $8.03 \%$.

2.2.7. Synthesis of 7-((6-(4-nitrophenyl)-7H-[1,2,4]triazolo [3,4-b][1,3,4]thiadiazin-3-yl)methoxy)-4-phenyl-2Hchromen-2-one (10)

Method A: A mixture of 7-[(4-amino-5-thioxo-4,5-dihydro- $1 H$ 1,2,4-triazol-3-yl)methoxy]-4-phenyl-2H-chromen-2-one (5) $(0.01 \mathrm{~mol}), \quad p$-nitrophenacyl bromide $(0.01 \mathrm{~mol})$ and triethylamine (TEA) $(0.2 \mathrm{~mL})$ in DMF $(15 \mathrm{~mL})$ was heated under reflux for $2 \mathrm{~h}$, then left to cool and poured onto crushed ice. The reaction mixture was acidified with dilute hydrochloric acid till $\mathrm{pH}=\sim 4$. The resulting solid was collected by filtration, washed with water and recrystallized from dioxane (Scheme 1). Color: Brown. Yield: $56 \%$. M.p.: $176-178{ }^{\circ} \mathrm{C}$. FT-IR (KBr, v, $\mathrm{cm}^{-1}$ ): 1712 (C=0). ${ }^{1} \mathrm{H}$ NMR (300 MHz, DMSO- $\left.d_{6}, \delta, \mathrm{ppm}\right): 6.76-$ $8.35(\mathrm{~m}, 12 \mathrm{H}, \mathrm{Ar}-\mathrm{H}), 6.23(\mathrm{~s}, 1 \mathrm{H}, \mathrm{H}-3), 5.22\left(\mathrm{~s}, 2 \mathrm{H}, \mathrm{OCH}_{2}\right), 4.81$ (s, $2 \mathrm{H}, \mathrm{CH}_{2}$ ). MS (EI, $\left.\mathrm{m} / z(\%)\right): 511\left(\mathrm{M}^{+}, 36\right)$. Anal.calcd. for $\mathrm{C}_{26} \mathrm{H}_{17} \mathrm{~N}_{5} \mathrm{O}_{5} \mathrm{~S}$ : C, 61.05; H, 3.35; N, 13.69. Found: C, 60.91; H, 3.23; N, $13.60 \%$.

Method B: To 7-\{[5-(2-(4-nitrophenyl)-2-oxoethylthio)1,3,4-oxadiazol-2-yl]methoxy\}-4-phenyl-2H-chromen-2-one (8) $(0.01 \mathrm{~mol})$ in ethanol $(20 \mathrm{~mL})$, hydrazine hydrate $(0.2 \mathrm{~mL})$ was added. The reaction mixture was heated under reflux for $3 \mathrm{~h}$, then left to cool. The resulting solid was collected by filtration, washed with water, and recrystallized from dioxane, to give compound 10.

2.2.8. Synthesis of 7-\{[4-(4-nitrobenzylideneamino)-5thioxo-4,5-dihydro-1H-1,2,4-triazol-3-yl]methoxy\}-4-phenyl2H-chromen-2-one (11)

A mixture of $p$-nitrobenzaldehyde $(0.01 \mathrm{~mol})$ and $7-[(4-$ amino-5-thioxo-4,5-dihydro-1H-1,2,4-triazol-3-yl)methoxy]-4phenyl-2H-chromen-2-one (5) $(0.01 \mathrm{~mol})$ in ethanol $(20 \mathrm{~mL})$ in the presence of acetic acid ( $1 \mathrm{~mL})$ was refluxed for $4 \mathrm{~h}$, then left to cool. The solid product was collected by filtration, washed with ethanol, dried and recrystallized from dioxane (Scheme 2). Color: Brown. Yield: $70 \%$. M.p.: $238-240^{\circ} \mathrm{C}$. FT-IR $\left(\mathrm{KBr}, v, \mathrm{~cm}^{-1}\right)$ : $3190(\mathrm{NH}), 1700(\mathrm{C}=0) .{ }^{1} \mathrm{H}$ NMR $\left(300 \mathrm{MHz}, \mathrm{DMSO}-d_{6}, \delta, \mathrm{ppm}\right)$ : $10.39\left(\mathrm{~s}, 1 \mathrm{H}, \mathrm{NH}\right.$ exchangeable with $\left.\mathrm{D}_{2} \mathrm{O}\right), 8.86(\mathrm{~s}, 1 \mathrm{H}, \mathrm{CH}=\mathrm{N})$, 7.03-8.38 (m, 12H, Ar-H), 6.28 (s, 1H, H-3), $5.40\left(\mathrm{~s}, 2 \mathrm{H}, \mathrm{OCH}_{2}\right)$. Anal. calcd. for $\mathrm{C}_{25} \mathrm{H}_{17} \mathrm{~N}_{5} \mathrm{O}_{5} \mathrm{~S}$ : C, 60.11; $\mathrm{H}, 3.43 ; \mathrm{N}, 14.02$. Found: C, 60.02; H, 3.30; N, 13.92\%. 


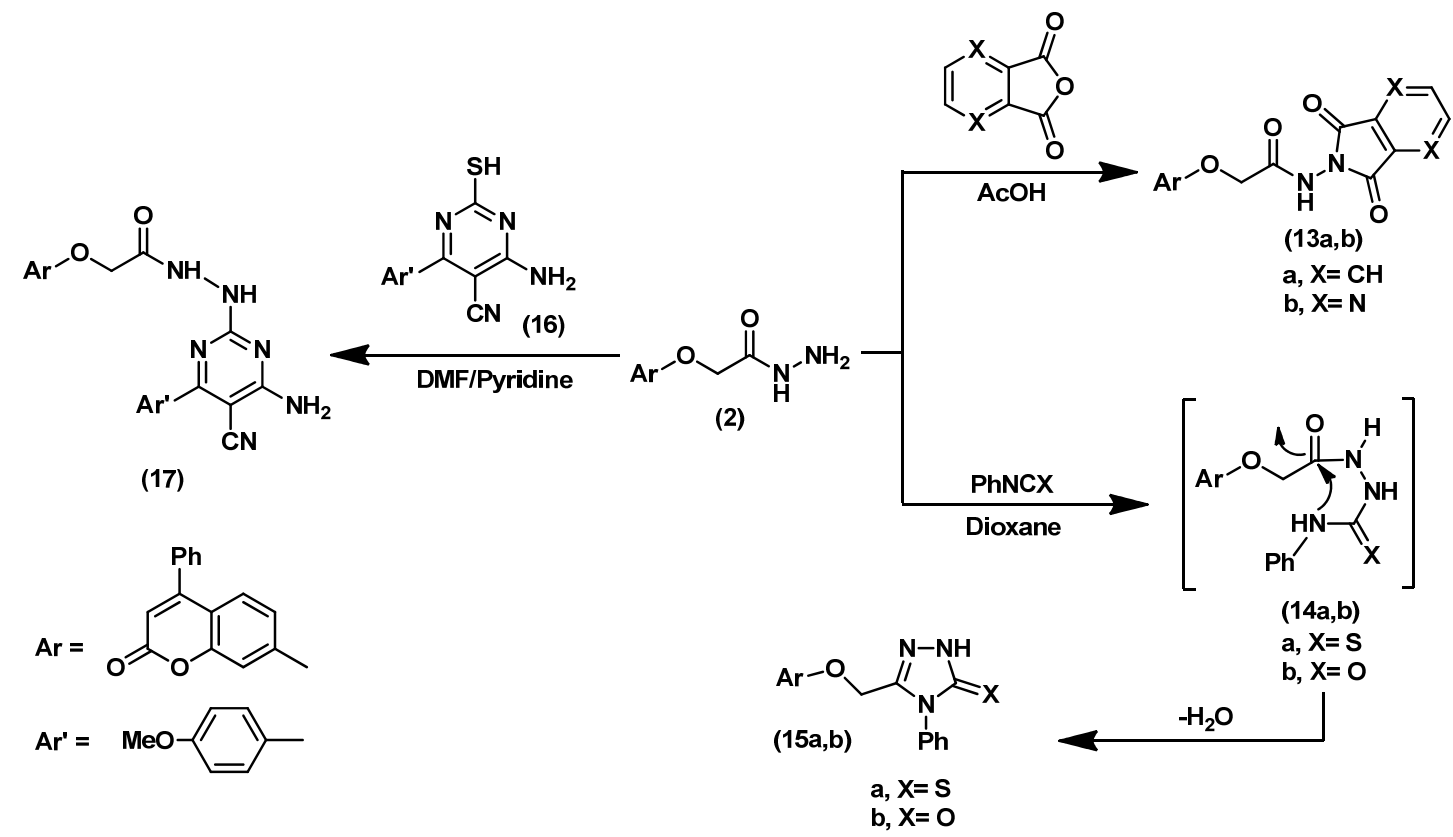

Scheme 3

2.2.9. General procedure for preparation of $N$-(substituted)2-(2-oxo-4-phenyl-2H-chromen-7-yloxy)acetamide (13a,b)

A mixture of 2-(2-oxo-4-phenyl-2H-chromen-7-yloxy)aceto hydrazide (2) $(0.01 \mathrm{~mol})$ and phthalic anhydride orpyrazine2,3-dicarboxylic anhydride $(0.01 \mathrm{~mol})$ in glacial acetic acid $(20$ $\mathrm{mL}$ ) was refluxed for $6 \mathrm{~h}$, then the reaction mixture left to cool. The white solid was filtered off and recrystallized from dioxane to give compounds 13a,b (Scheme 3).

$\mathrm{N}$-(1,3-Dioxoisoindolin-2-yl)-2-(2-oxo-4-phenyl-2H-chromen7-yloxy)-acetamide (13a): Color: White. Yield: 80\%. M.p.: 262$264^{\circ} \mathrm{C}$. FT-IR (KBr, $\left.v, \mathrm{~cm}^{-1}\right): 3176(\mathrm{NH}), 1715,1708$ (C=0). ${ }^{1} \mathrm{H}$ NMR (300 MHz, DMSO- $\left.d_{6}, \delta, \mathrm{ppm}\right): 11.09(\mathrm{~s}, 1 \mathrm{H}, \mathrm{NH}), 7.04-8.01$ (m, 12H, Ar-H), 6.29 (s, 1H, H-3), $5.01\left(\mathrm{~s}, 2 \mathrm{H}, \mathrm{OCH}_{2}\right)$. MS (EI, $m / z$ (\%)): $440\left(\mathrm{M}^{+}, 20\right)$. Anal. calcd. for $\mathrm{C}_{25} \mathrm{H}_{16} \mathrm{~N}_{2} \mathrm{O}_{6}$ : C, 68.18; $\mathrm{H}, 3.66$; $\mathrm{N}, 6.36$. Found: C, 68.10; $\mathrm{H}, 3.58 ; \mathrm{N}, 6.29 \%$.

$\mathrm{N}$-(5,7-Dioxo-5H-pyrrolo[3,4-b]pyrazin-6(7H)-yl)-2-(2-oxo-4phenyl-2H-chromen-7-yloxy)acetamide (13b): Color: White. Yield: $82 \%$. M.p.: $158-160{ }^{\circ} \mathrm{C}$. FT-IR $\left(\mathrm{KBr}, v, \mathrm{~cm}^{-1}\right): 3276(\mathrm{NH})$, 1720, 1714 (C=0). ${ }^{1} \mathrm{H}$ NMR (300 MHz, DMSO- $\left.d_{6}, \delta, \mathrm{ppm}\right): 10.78$ (s, $1 \mathrm{H}, \mathrm{NH}), 8.76,8.90(\mathrm{~d}, 2 \mathrm{H}, 2 \mathrm{CH}$ pyrazine), $6.93-7.55(\mathrm{~m}, 8 \mathrm{H}$, Ar-H), 6.24 (s, $1 \mathrm{H}, \mathrm{H}-3), 4.84\left(\mathrm{~s}, 2 \mathrm{H}, \mathrm{OCH}_{2}\right) .{ }^{13} \mathrm{CNMR}(300 \mathrm{MHz}$, DMSO- $\left.d_{6}, \delta, \mathrm{ppm}\right): 166.06,162.02,160.75,159.84,155.19$, $154.99,147.96,144.00,143.67,143.52,134.87,129.62,128.81$, $128.35,127.76,120.79,112.93,112.35,111.68,102.16$ and 66.36. MS (EI, $m / z(\%)): 442\left(\mathrm{M}^{+}, 50\right)$. Anal. calcd. for $\mathrm{C}_{23} \mathrm{H}_{14} \mathrm{~N}_{4} \mathrm{O}_{6}$ : C, 62.45; $\mathrm{H}, 3.19$; N, 12.66. Found: $\mathrm{C}, 62.38 ; \mathrm{H}, 3.10$; $\mathrm{N}, 12.52 \%$.

2.2.10. General procedure for the preparation of 4-phenyl-7((4-phenyl-5-substituted-4,5-dihydro-1H-1,2,4-triazol-3-yl) methoxy)-2H-chromen-2-one (15a,b)

A mixture of 2-(2-oxo-4-phenyl-2H-chromen-7-yloxy)aceto hydrazide (2) $(0.01 \mathrm{~mol})$ and phenylisothiocyanate or phenyliso-cyanate $(0.01 \mathrm{~mol})$ in dioxane $(15 \mathrm{~mL})$ was refluxed for $3 \mathrm{~h}$, the resulting solid on heating was collected by filtration, washed with ethanol several times and recrystallized from DMF to give compounds 15a,b (Scheme 3 ).

4-Phenyl-7-((4-phenyl-5-thioxo-4,5-dihydro-1H-1,2,4-triazol3-yl)methoxy)-2H-chromen-2-one (15a): Color: White. Yield:
76\%. M.p.: 224-226 ${ }^{\circ} \mathrm{C}$. FT-IR (KBr, v, cm $\left.{ }^{-1}\right): 3345(\mathrm{NH}), 1720$ $(\mathrm{C}=0) .{ }^{1} \mathrm{H}$ NMR (300 MHz, DMSO- $\left.d_{6}, \delta, \mathrm{ppm}\right): 14.08(\mathrm{~s}, 1 \mathrm{H}, \mathrm{NH})$, 6.78-7.56 (m, 13H, Ar-H), 6.24 (s, 1H, H-3), $5.13\left(\mathrm{~s}, 2 \mathrm{H}, \mathrm{OCH}_{2}\right)$. MS (EI, $m / z(\%)$ ): $427\left(\mathrm{M}^{+}, 10\right)$. Anal. calcd. for $\mathrm{C}_{24} \mathrm{H}_{17} \mathrm{~N}_{3} \mathrm{O}_{3} \mathrm{~S}$ : C, 67.43; H, 4.01; N 9.83. Found: C, 67.37; H, 3.93; N, 9.75\%.

3-((2-Oxo-4-phenyl-2H-chromen-7-yloxy)methyl)-4-phenyl1H-1,2,4-triazol-5(4H)-one (15b): Color: White. Yield: 82\%. M.p.: 220-222 ${ }^{\circ} \mathrm{C}$. FT-IR (KBr, v, cm-1): $3302(\mathrm{NH}), 1692$ (C=0). ${ }^{1} \mathrm{H}$ NMR (300 MHz, DMSO- $\left.d_{6}, \delta, \mathrm{ppm}\right): 10.06(\mathrm{~s}, 1 \mathrm{H}, \mathrm{NH}), 6.95-$ $8.74(\mathrm{~m}, 13 \mathrm{H}, \mathrm{Ar}-\mathrm{H}), 6.27$ (s, 1H, H-3), $4.78\left(\mathrm{~s}, 2 \mathrm{H}, \mathrm{OCH}_{2}\right) . \mathrm{MS}$ (EI, $m / z(\%)$ ): $411\left(\mathrm{M}^{+}, 75\right)$. Anal. calcd. for $\mathrm{C}_{24} \mathrm{H}_{17} \mathrm{~N}_{3} \mathrm{O}_{4}$ : C, 70.07; H, 4.16; N, 10.21. Found: C, 69.89; H, 4.08; N, 10.10\%.

2.2.11. Synthesis of $N$ '-(4-amino-5-cyano-6-(4-methoxy phenyl) pyrimidin-2-yl)-2-(2-oxo-4-phenyl-2H-chromen-7yloxy) acetohydrazide (17)

A mixture of 2-(2-oxo-4-phenyl-2H-chromen-7-yloxy)aceto hydrazide (2) (0.01 mol),4-amino-2-mercapto-6-(4-methoxyphenyl)pyrimidine-5-carbonitrile (16) $(0.01 \mathrm{~mol})$ and pyridine $(0.2 \mathrm{~mL})$ in DMF $(15 \mathrm{~mL})$ was heated under reflux for $4 \mathrm{~h}$, then left to cool and poured onto crushed ice. The reaction mixture was acidified with dilute hydrochloric acid till $\mathrm{pH}=\sim 4$. The resulting solid was collected by filtration, washed with water and recrystallized from dioxane (Scheme 3). Color: Yellow. Yield: 92\%. M.p.: 98-100 ${ }^{\circ} \mathrm{C}$. FT-IR (KBr, v, cm$\left.{ }^{-1}\right): 3325\left(\mathrm{NH}_{2}\right)$, $3230(\mathrm{NH}), 2200(\mathrm{CN}), 1712(\mathrm{C}=0) .{ }^{1} \mathrm{H}$ NMR $(300 \mathrm{MHz}$, DMSO$\left.d_{6}, \delta, \mathrm{ppm}\right): 10.03(\mathrm{~s}, 1 \mathrm{H}, \mathrm{NH}), 10.29(\mathrm{~s}, 1 \mathrm{H}, \mathrm{NH}), 8.02$ (s, 2H, $\left.\mathrm{NH}_{2}\right), 7.01-7.81(\mathrm{~m}, 12 \mathrm{H}, \mathrm{Ar}-\mathrm{H}), 6.24(\mathrm{~s}, 1 \mathrm{H}, \mathrm{H}-3), 4.74(\mathrm{~s}, 2 \mathrm{H}$, $\left.\mathrm{OCH}_{2}\right), 3.79$ (s, $3 \mathrm{H}, \mathrm{OCH}_{3}$ ). Anal. calcd. for $\mathrm{C}_{29} \mathrm{H}_{22} \mathrm{~N}_{6} \mathrm{O}_{5}: \mathrm{C}, 65.16$; H, 4.15; N, 15.72. Found: C, 65.08; H, 4.07; N, 15.65\%.

\subsubsection{Synthesis of 7-[(6-benzyl-[1,2,4]triazolo[3,4-a]} phthalazin-3-yl)methoxy]-4-phenyl-2H-chromen-2-one (20)

A mixture of 2-(2-oxo-4-phenyl-2H-chromen-7-yloxy) aceto hydrazide (2) (0.01 mol) and 4-benzyl-1-chlorophthalazine (18) $(0.01 \mathrm{~mol})$ in dry dioxane $(10 \mathrm{~mL})$ and a catalytic amount of pyridine was refluxed for $4 \mathrm{~h}$. The solid product which obtained after cooling was collected and recrystallized from dioxane to give compound $\mathbf{2 0}$ (Scheme 4). 


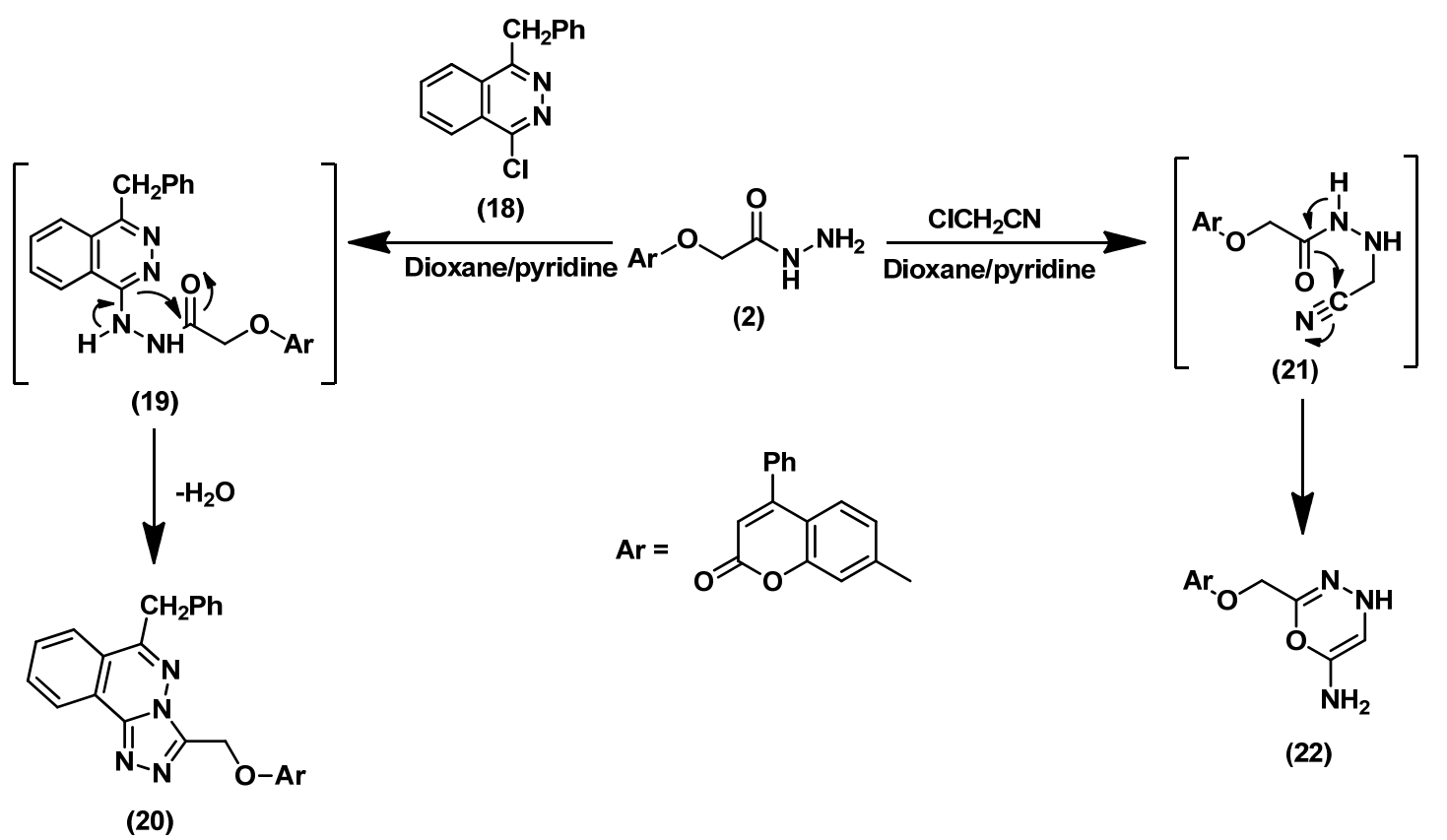

Scheme 4

Color: Yellow. Yield: $84 \%$. M.p.: $170-172{ }^{\circ} \mathrm{C}$. FT-IR (KBr, v, $\mathrm{cm}^{-1}$ ): 3012 (CH-aromatic), 2902 (CH-aliphatic), $1712(\mathrm{C}=0) .{ }^{1} \mathrm{H}$ NMR (300 MHz, DMSO- $\left.d_{6}, \delta, \mathrm{ppm}\right): 6.96-8.26(\mathrm{~m}, 17 \mathrm{H}, \mathrm{Ar}-\mathrm{H})$, $6.25(\mathrm{~s}, 1 \mathrm{H}, \mathrm{H}-3), 4.63\left(\mathrm{~s}, 2 \mathrm{H}, \mathrm{OCH}_{2}\right), 4.35\left(\mathrm{~s}, 2 \mathrm{H}, \mathrm{CH}_{2}\right)$. MS (EI, $m / z(\%)): 510\left(\mathrm{M}^{+}, 29\right)$. Anal. calcd. for $\mathrm{C}_{32} \mathrm{H}_{22} \mathrm{~N}_{4} \mathrm{O}_{3}$ : C, 75.28; $\mathrm{H}$, $4.34 ; \mathrm{N}, 10.97$. Found: $\mathrm{C}, 75.20 ; \mathrm{H}, 4.23 ; \mathrm{N}, 10.90 \%$.

\subsubsection{Synthesis of 7-[(6-amino-4H-1,3,4-oxadiazin-2-yl) methoxy]-4-phenyl-2H-chromen-2-one (22)}

A mixture of 2-(2-oxo-4-phenyl-2H-chromen-7-yloxy)aceto hydrazide (2) $(0.01 \mathrm{~mol})$ and chloroacetonitrile $(0.01 \mathrm{~mol})$ in dry dioxane $(10 \mathrm{~mL})$ and a catalytic amount of pyridine was refluxed for $3 \mathrm{~h}$, then the reaction mixture left to cool. The solid product was filtered off, washed with ethanol several times and recrystallized from dioxane (Scheme 4). Color: White. Yield: 56\%. M.p.: $240-242{ }^{\circ} \mathrm{C}$. FT-IR (KBr, v, cm-1): $3245\left(\mathrm{NH}_{2}\right), 3175$ $(\mathrm{NH}), 1712(\mathrm{C}=0) .{ }^{1} \mathrm{H}$ NMR (300 MHz, DMSO- $\left.d_{6}, \delta, \mathrm{ppm}\right): 10.29$ $(\mathrm{s}, 1 \mathrm{H}, \mathrm{NH}), 7.07-7.53\left(\mathrm{~m}, 11 \mathrm{H}, \mathrm{Ar}-\mathrm{H}, \mathrm{CH}-\right.$ oxadiazin and $\left.\mathrm{NH}_{2}\right)$, 6.23 (s, 1H, H-3), $4.76\left(\mathrm{~s}, 2 \mathrm{H}, \mathrm{OCH}_{2}\right)$. MS (EI, $\left.m / z(\%)\right): 349\left(\mathrm{M}^{+}\right.$, 16). Anal. calcd. for $\mathrm{C}_{19} \mathrm{H}_{15} \mathrm{~N}_{3} \mathrm{O}_{4}$ : C, 65.32; H, 4.33; N, 12.03 . Found: C, 65.19; $\mathrm{H}, 4.12 ; \mathrm{N}, 11.83 \%$.

\subsubsection{Synthesis of 7-[(4,5-dihydro-1H-imidazol-2-yl) methoxy]-4-phenyl-2H-chromen-2-one (24)}

A mixture of 2-(2-oxo-4-phenyl-2H-chromen-7-yloxy) acetic acid (23) $(0.01 \mathrm{~mol})$ and ethylenediamine $(0.01 \mathrm{~mol})$ was heated without solvent at $180-200{ }^{\circ} \mathrm{C}$ for $30 \mathrm{~min}$. Then the mixture was left to cool. The solid product that formed was recrystallized from dioxane (Scheme 5). Color: Brown. Yield: 58\%. M.p.: 266-268 ${ }^{\circ} \mathrm{C}$. FT-IR $\left(\mathrm{KBr}, v, \mathrm{~cm}^{-1}\right)$ : $3376(\mathrm{NH}), 1712$ $(\mathrm{C}=0) .{ }^{1} \mathrm{H}$ NMR $\left(300 \mathrm{MHz}, \mathrm{DMSO}-d_{6}, \delta, \mathrm{ppm}\right): 8.24(\mathrm{~s}, 1 \mathrm{H}, \mathrm{NH})$, 6.91-7.56 (m, 8H, Ar-H), 6.22 (s, 1H, H-3), 4.59 (s, 2H, OCH $)$, $3.28\left(\mathrm{~s}, 4 \mathrm{H}, 2 \mathrm{CH}_{2}\right)$. MS (EI, $\left.m / z(\%)\right): 320\left(\mathrm{M}^{+}, 17\right)$. Anal. calcd. for $\mathrm{C}_{19} \mathrm{H}_{16} \mathrm{~N}_{2} \mathrm{O}_{3}$ : C, 71.24; $\mathrm{H}, 5.03$; N, 8.74. Found: C, 71.17; $\mathrm{H}$, $4.91 ; \mathrm{N}, 8.69 \%$.

2.2.15. General procedure for preparation of 7-(substituted2-ylmethoxy)-4-phenyl-2H-chromen-2-one (25a-c)
A mixture of 2-(2-oxo-4-phenyl-2H-chromen-7-yloxy) acetic acid (23) (0.01 mol) and 1,2-phenylenediamine, or 2aminophenol, or 2-aminothiophenol $(0.01 \mathrm{~mol})$ was heated without solvent at $180-200^{\circ} \mathrm{C}$ for $30 \mathrm{~min}$. Then the mixture was left to cool. The solid products that formed were recrystallized from dioxane to give compounds 25a-c (Scheme 5).

7-[(1H-Benzo[d]imidazol-2-yl)methoxy]-4-phenyl-2Hchromen-2-one (25a): Color: Brown. Yield: 64\%. M.p.: 242-244 ${ }^{\circ} \mathrm{C}$. FT-IR (KBr, v, cm-1): $3320(\mathrm{NH}), 1706(\mathrm{C}=0) .{ }^{1} \mathrm{H}$ NMR $(300$ MHz, DMSO- $\left.d_{6}, \delta, \mathrm{ppm}\right): 12.72(\mathrm{~s}, 1 \mathrm{H}, \mathrm{NH}), 7.05-7.65(\mathrm{~m}, 12 \mathrm{H}$, $\mathrm{Ar}-\mathrm{H}), 6.25(\mathrm{~s}, 1 \mathrm{H}, \mathrm{H}-3), 5.46\left(\mathrm{~s}, 2 \mathrm{H}, \mathrm{OCH}_{2}\right)$. Anal. calcd. for $\mathrm{C}_{23} \mathrm{H}_{16} \mathrm{~N}_{2} \mathrm{O}_{3}$ : C, 74.99; H, 4.38; N, 7.60. Found: C, 74.91; H, 4.30; $\mathrm{N}, 7.52 \%$.

7-(Benzo[d]oxazol-2-ylmethoxy)-4-phenyl-2H-chromen-2one (25b): Color: Reddish brown. Yield: $52 \%$. M.p.: $170-172{ }^{\circ} \mathrm{C}$. FT-IR (KBr, $\left.v, \mathrm{~cm}^{-1}\right): 1730(\mathrm{C}=0) .{ }^{1} \mathrm{H}$ NMR $\left(300 \mathrm{MHz}\right.$, DMSO- $d_{6}$, $\delta, \mathrm{ppm}):$ 7.05-7.80 (m, 12H, Ar-H), $6.26(\mathrm{~s}, 1 \mathrm{H}, \mathrm{H}-3), 5.63(\mathrm{~s}, 2 \mathrm{H}$, $\mathrm{OCH}_{2}$ ). MS (EI, $m / z(\%)$ ): $369\left(\mathrm{M}^{+}, 32\right)$. Anal. calcd. for $\mathrm{C}_{23} \mathrm{H}_{15} \mathrm{NO}_{4}$ : C, 74.79; H, 4.09; N, 3.79. Found: C, 74.68; H, 4.00; N, $3.68 \%$.

7-(Benzo[d] thiazol-2-ylmethoxy)-4-phenyl-2H-chromen-2one (25c): Color: Yellow. Yield: 78\%. M.p.: $138^{\circ} \mathrm{C}$. FT-IR (KBr, $v$, $\left.\mathrm{cm}^{-1}\right): 1716(\mathrm{C}=0)$. ${ }^{1} \mathrm{H}$ NMR (300 MHz, DMSO- $\left.d_{6}, \delta, \mathrm{ppm}\right):$ : 7.08$8.15(\mathrm{~m}, 12 \mathrm{H}, \mathrm{Ar}-\mathrm{H}), 6.27(\mathrm{~s}, 1 \mathrm{H}, \mathrm{H}-3), 5.76\left(\mathrm{~s}, 2 \mathrm{H}, \mathrm{OCH}_{2}\right)$. MS (EI, $m / z(\%)): 385\left(\mathrm{M}^{+}, 26\right)$. Anal. calcd. for $\mathrm{C}_{23} \mathrm{H}_{15} \mathrm{NO}_{3} \mathrm{~S}$ : C, 71.67; H, 3.92; N, 3.63. Found: C, 71.58; H, 3.83; N, 3.57\%.

\subsubsection{Synthesis of 2-(2-oxo-4-phenyl-2H-chromen-7-yloxy) acetyl chloride (26)}

A mixture of 2-(2-oxo-4-phenyl-2 $\mathrm{H}$-chromen-7-yloxy)acetic acid (23) $(0.01 \mathrm{~mol})$ and thionyl chloride $(15 \mathrm{~mL})$ was fused for $1 \mathrm{~h}$. Excess of thionyl chloride removed under pressure to obtain the pure acyl chloride (Scheme 5). Color: Pale yellow. Yield: 95\%. M.p.: $140-142{ }^{\circ} \mathrm{C}$. FT-IR (KBr, v, $\left.\mathrm{cm}^{-1}\right): 1700$ (C=0). ${ }^{1} \mathrm{H}$ NMR $\left(300 \mathrm{MHz}, \mathrm{DMSO}-d_{6}, \delta, \mathrm{ppm}\right): 6.76-7.58(\mathrm{~m}, 8 \mathrm{H}, \mathrm{Ar}-\mathrm{H})$, 6.15 (s, $1 \mathrm{H}, \mathrm{H}-3), 4.84\left(\mathrm{~s}, 2 \mathrm{H}, \mathrm{OCH}_{2}\right)$. MS (EI, $\left.m / z(\%)\right): 314\left(\mathrm{M}^{+}\right.$, 25). Anal. calcd. for $\mathrm{C}_{17} \mathrm{H}_{11} \mathrm{ClO}_{4}$ : $\mathrm{C}, 64.88 ; \mathrm{H}, 3.52$. Found: $\mathrm{C}$, $64.80 ; \mathrm{H}, 3.44 \%$. 


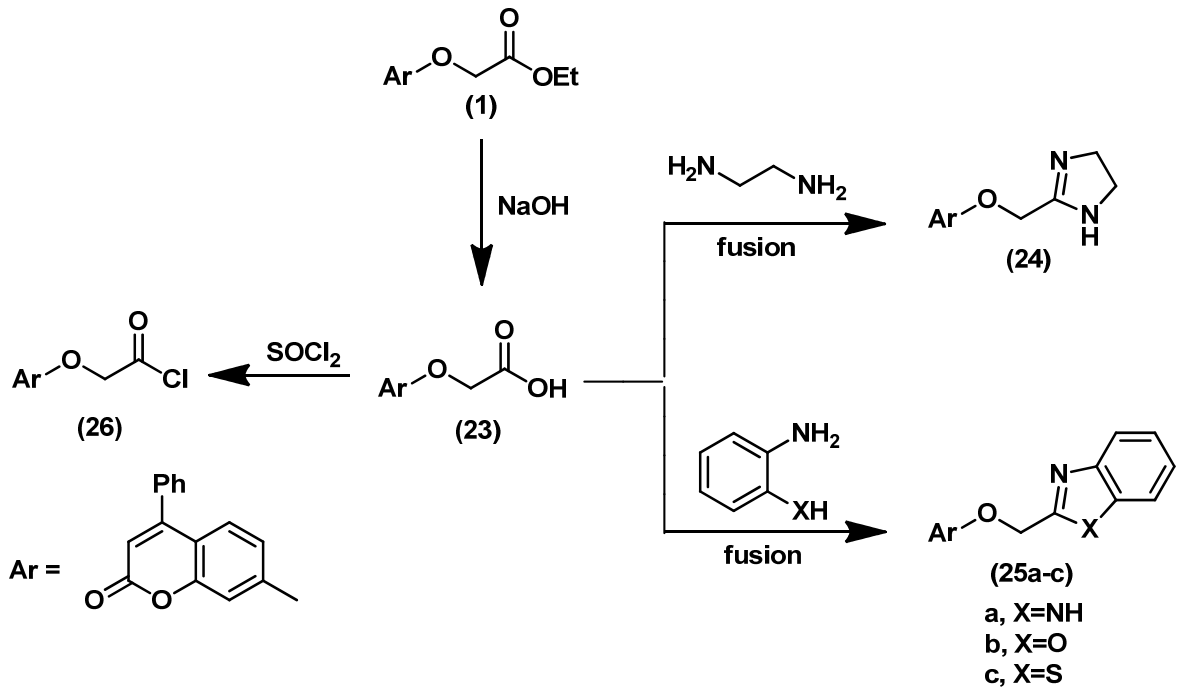

Scheme 5

\subsubsection{General procedure for preparation of $\mathrm{N}$-(4-acetyl phenyl)-2-(2-oxo-4-phenyl-2H-chromen-7-yloxy)acetamide, 2-\{4-[2-(2-oxo-4-phenyl-2H-chromen-7-yloxy)acetamido] phenyl] acetic acid and 4-[2-(2-0xo-4-phenyl-2H-chromen-7- yloxy)acetamido]benzoic acid (27a-c)}

A mixture of 2-(2-oxo-4-phenyl-2H-chromen-7-yloxy)acetyl chloride (26) $(0.01 \mathrm{~mol})$ and aniline derivatives namely (4amino-acetophenone, 4-aminophenylacetic acid and 4aminobenzoic acid) $(0.01 \mathrm{~mol})$ in dry dioxane $(10 \mathrm{~mL})$ and a catalytic amount of pyridine was refluxed for $1 \mathrm{~h}$, the resulting solid on heating was collected by filtration, washed with ethanol several times and recrystallized from DMF to give compounds 27a-c (Scheme 6).

$\mathrm{N}$-(4-Acetylphenyl)-2-(2-oxo-4-phenyl-2H-chromen-7-yloxy) acetamide (27a): Color: White. Yield: $97 \%$. M.p.: $222-224{ }^{\circ} \mathrm{C}$. FT-IR (KBr, v, cm $\left.{ }^{-1}\right): 3344(\mathrm{NH}), 1718,1682(\mathrm{C}=0) .{ }^{1} \mathrm{H}$ NMR (300 MHz, DMSO- $\left.d_{6}, \delta, \mathrm{ppm}\right): 10.48(\mathrm{~s}, 1 \mathrm{H}, \mathrm{NH}), 7.01-7.96(\mathrm{~m}$, $12 \mathrm{H}, \mathrm{Ar}-\mathrm{H}), 6.26(\mathrm{~s}, 1 \mathrm{H}, \mathrm{H}-3), 4.91\left(\mathrm{~s}, 2 \mathrm{H}, \mathrm{OCH}_{2}\right), 2.48(\mathrm{~s}, 3 \mathrm{H}$, $\mathrm{CH}_{3}$ ). Anal. calcd. for $\mathrm{C}_{25} \mathrm{H}_{19} \mathrm{NO}_{5}$ : C, 72.63; $\mathrm{H}, 4.63$; N, 3.39. Found: C, 72.51; $\mathrm{H}, 4.58 ; \mathrm{N}, 3.28 \%$.

2-\{4-[2-(2-Oxo-4-phenyl-2H-chromen-7-yloxy)acetamido] phenyl\}acetic acid (27b): Color: Yellow. Yield: 90\%. M.p.: 216$218^{\circ} \mathrm{C}$. FT-IR (KBr, $\left.v, \mathrm{~cm}^{-1}\right): 3432(\mathrm{OH}), 3334(\mathrm{NH}), 1720(\mathrm{C}=0)$. ${ }_{1}^{1} \mathrm{H}$ NMR (300 MHz, DMSO- $d_{6}, \delta, \mathrm{ppm}$ ): 12.22 (s, $1 \mathrm{H}, \mathrm{OH}$ exchangeable with $\left.\mathrm{D}_{2} \mathrm{O}\right), 10.12(\mathrm{~s}, 1 \mathrm{H}, \mathrm{NH}$ exchangeable with $\left.\mathrm{D}_{2} \mathrm{O}\right)$, 6.80-7.62 (m, $\left.12 \mathrm{H}, \mathrm{Ar}-\mathrm{H}\right), 6.25(\mathrm{~s}, 1 \mathrm{H}, \mathrm{H}-3), 4.85(\mathrm{~s}, 2 \mathrm{H}$, $\left.\mathrm{OCH}_{2}\right), 3.56\left(\mathrm{~s}, 2 \mathrm{H}, \mathrm{CH}_{2}\right)$. MS (EI, $\left.m / z(\%)\right): 429\left(\mathrm{M}^{+}, 10\right)$. Anal. calcd. for $\mathrm{C}_{25} \mathrm{H}_{19} \mathrm{NO}_{6}$ : C, 69.92; H, 4.46; N, 3.26. Found: C, 69.84; $\mathrm{H}, 4.38$; N, 3.18\%

4-(2-(2-Oxo-4-phenyl-2H-chromen-7-yloxy)acetamido) benzoic acid (27c): Color: White. Yield: 95\%. M.p.: 316-318 ${ }^{\circ} \mathrm{C}$. FT-IR (KBr, v, cm-1): $3435(\mathrm{OH}), 3352(\mathrm{NH}), 1698(\mathrm{C}=0) .{ }^{1} \mathrm{H}$ NMR (300 MHz, DMSO- $\left.d_{6}, \delta, \mathrm{ppm}\right): 12.71$ (s, $1 \mathrm{H}, \mathrm{OH}$ exchangeable with $\left.\mathrm{D}_{2} \mathrm{O}\right), 10.45(\mathrm{~s}, 1 \mathrm{H}, \mathrm{NH}$ exchangeable with $\left.\mathrm{D}_{2} \mathrm{O}\right), 7.01-7.93(\mathrm{~m}, 12 \mathrm{H}, \mathrm{Ar}-\mathrm{H}), 6.26(\mathrm{~s}, 1 \mathrm{H}, \mathrm{H}-3), 4.91(\mathrm{~s}, 2 \mathrm{H}$, $\mathrm{OCH}_{2}$ ). MS (EI, $\left.m / z(\%)\right): 415\left(\mathrm{M}^{+}, 16\right)$. Anal. calcd. for $\mathrm{C}_{24} \mathrm{H}_{17} \mathrm{NO}_{6}$ : C, 69.39; H, 4.12; N, 3.37. Found: C, 69.30; H, 4.06; N, $3.26 \%$.

\subsubsection{Synthesis of 4-[2-(2-oxo-4-phenyl-2H-chromen-7-} yloxy)acetamido]benzenesulfonic acid (28)

A mixture of 2-(2-oxo-4-phenyl-2H-chromen-7-yloxy)acetyl chloride (26) $(0.01 \mathrm{~mol})$ and 4-aminobenzenesulfonic acid $(0.01 \mathrm{~mol})$ in dry dioxane $(10 \mathrm{~mL})$ and a catalytic amount of pyridine was refluxed for $1 \mathrm{~h}$, the resulting solid on heating was collected by filtration, washed with ethanol several times and recrystallized from DMF to give compound $\mathbf{2 8}$ (Scheme 6) Color: White. Yield: $70 \%$. M.p.: $344-346^{\circ} \mathrm{C}$. FT-IR $\left(\mathrm{KBr}, v, \mathrm{~cm}^{-1}\right)$ : $3430(\mathrm{OH}), 3235(\mathrm{NH}), 1700(\mathrm{C}=0) .{ }^{1} \mathrm{H}$ NMR $\left(300 \mathrm{MHz}\right.$, DMSO- $d_{6}$, $\delta, \mathrm{ppm}): 14.01(\mathrm{~s}, 1 \mathrm{H}, \mathrm{OH}), 9.16(\mathrm{~s}, 1 \mathrm{H}, \mathrm{NH}), 7.04-7.70(\mathrm{~m}, 12 \mathrm{H}$, Ar-H), 6.26 (s, 1H, H-3), 4.92 (s, $2 \mathrm{H}, \mathrm{OCH}_{2}$ ). MS (EI, $m / z(\%)$ ): $451\left(\mathrm{M}^{+}, 22\right)$. Anal. calcd. for $\mathrm{C}_{23} \mathrm{H}_{17} \mathrm{NO}_{7} \mathrm{~S}: \mathrm{C}, 61.19 ; \mathrm{H}, 3.80 ; \mathrm{N}$, 3.10. Found: C, 61.08; H, 3.73; N, 3.02\%.

2.2.19. General procedure for preparation of 2-[2-(2-oxo-4phenyl-2H-chromen-7-yloxy)acetamido] benzoic acid and Methyl-2-[2-(2-oxo-4-phenyl-2H-chromen-7-yloxy) acetamido] benzoate $(29 a, b)$

A mixture of 2-(2-oxo-4-phenyl-2H-chromen-7-yloxy)acetyl chloride (26) $(0.01 \mathrm{~mol})$ and anthranilic acid or methyl anthranilate $(0.01 \mathrm{~mol})$ in dry dioxane $(10 \mathrm{~mL})$ and a catalytic amount of pyridine was refluxed for $1 \mathrm{~h}$, the resulting solid on heating was collected by filtration, washed with ethanol several times and recrystallized from DMF to give compounds 29a,b (Scheme 6).

2-[2-(2-Oxo-4-phenyl-2H-chromen-7-yloxy)acetamido] benzoicacid (29a): Color: White. Yield: $90 \%$. M.p.: $280-282^{\circ} \mathrm{C}$. FT-IR (KBr, $\left.v, \mathrm{~cm}^{-1}\right)$ : 3258(NH), 1732, $1686(\mathrm{C}=0) .{ }^{1} \mathrm{H}$ NMR $(300$ MHz, DMSO- $\left.d_{6}, \delta, \mathrm{ppm}\right): 13.90(\mathrm{~s}, 1 \mathrm{H}, \mathrm{OH}), 12.12(\mathrm{~s}, 1 \mathrm{H}, \mathrm{NH})$, 7.07-8.68 (m, 12H, Ar-H), 6.28 (s, 1H, H-3), $4.90\left(\mathrm{~s}, 2 \mathrm{H}, \mathrm{OCH}_{2}\right)$. MS (EI, $m / z(\%)): 415\left(\mathrm{M}^{+}, 10\right)$. Anal. calcd. for $\mathrm{C}_{24} \mathrm{H}_{17} \mathrm{NO}_{6}$ : C, 69.39; H, 4.12; N, 3.37. Found: C, 69.30; H, 4.05; N, 3.27\%.

Methyl-2-[2-(2-oxo-4-phenyl-2H-chromen-7-yloxy) acetamidolbenzoate (29b): Color: White. Yield: 87\%. M.p.: 202-204 ${ }^{\circ} \mathrm{C}$. FT-IR (KBr, v, cm-1): 3244(NH), 1726, $1694(\mathrm{C}=0) .{ }^{1} \mathrm{H}$ NMR (300 MHz, DMSO- $\left.d_{6}, \delta, \mathrm{ppm}\right): 11.65(\mathrm{~s}, 1 \mathrm{H}, \mathrm{NH}), 7.10-8.58(\mathrm{~m}$, $12 \mathrm{H}, \mathrm{Ar}-\mathrm{H}), 6.28(\mathrm{~s}, 1 \mathrm{H}, \mathrm{H}-3), 4.90\left(\mathrm{~s}, 2 \mathrm{H}, \mathrm{OCH}_{2}\right), 3.95(\mathrm{~s}, 3 \mathrm{H}$, $\mathrm{OCH}_{3}$ ). Anal. calcd. for $\mathrm{C}_{25} \mathrm{H}_{19} \mathrm{NO}_{6}$ : C, 69.92; $\mathrm{H}, 4.46$; $\mathrm{N}, 3.26$. Found: C, 69.85; H, 4.38; N, 3.19\%.

\subsubsection{Synthesis of $N, N^{\prime}$-bis [2-(2-oxo-4-phenyl-2H-chromen-} 7-yloxy) acetyl] hydrazine (30)

A mixture of 2-(2-oxo-4-phenyl-2H-chromen-7-yloxy) acetyl chloride (26) $(0.01 \mathrm{~mol})$ and 2-(2-oxo-4-phenyl- $2 \mathrm{H}$ chromen-7-yloxy) acetohydrazide (2) $(0.01 \mathrm{~mol})$ in dry dioxane $(10 \mathrm{~mL})$ and a catalytic amount of pyridine was refluxed for $2 \mathrm{~h}$. 


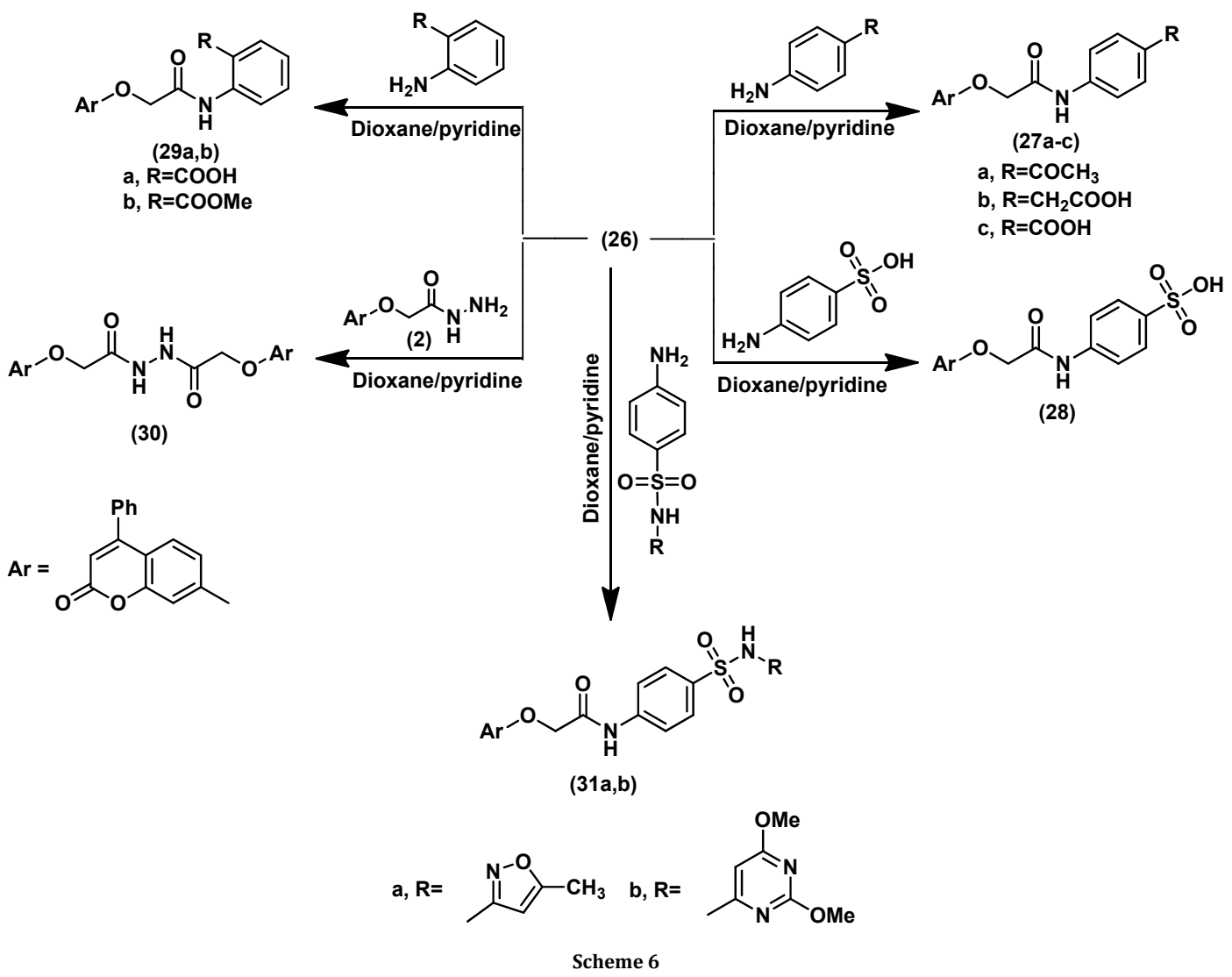

The precipitate formed was filtered off hot, washed with ethanol several times, dried and recrystallized from DMF to give compound 30. Color: White. Yield: $80 \%$. M.p.: $246-248{ }^{\circ} \mathrm{C}$. FT-IR $\left(\mathrm{KBr}, v, \mathrm{~cm}^{-1}\right): 3220(\mathrm{NH}), 1715(\mathrm{C}=0) .{ }^{1} \mathrm{H}$ NMR $(300 \mathrm{MHz}$, DMSO- $\left.d_{6}, \delta, p p m\right): 10.29(\mathrm{~s}, 2 \mathrm{H}, 2 \mathrm{NH}), 6.99-7.58(\mathrm{~m}, 16 \mathrm{H}, \mathrm{Ar}-\mathrm{H})$, $6.26(\mathrm{~s}, 2 \mathrm{H}, 2 \mathrm{H}-3), 4.79\left(\mathrm{~s}, 4 \mathrm{H}, 2 \mathrm{CH}_{2}\right.$ ). MS (EI, $\left.\mathrm{m} / z(\%)\right): 588$ $\left(\mathrm{M}^{+}, 27\right)$. Anal. calcd. for $\mathrm{C}_{34} \mathrm{H}_{24} \mathrm{~N}_{2} \mathrm{O}_{8}$ : C, 69.38; $\mathrm{H}, 4.11 ; \mathrm{N}, 4.76$. Found: C, 69.28; H, 4.03; N, 4.69\%.

\subsubsection{General procedure for preparation of $\mathrm{N}-\{4-[\mathrm{N}$ - (substituted) sulfamoyl] phenyl\}-2-(2-oxo-4-phenyl-2H- chromen-7-yloxy) acetamide $(31 a, b)$.}

A mixture of 2-(2-oxo-4-phenyl-2H-chromen-7-yloxy) acetyl chloride (26) $(0.01 \mathrm{~mol})$ and benzenesulfonamide derivatives namely (4-amino- $N$-(5-methylisoxazol-3-yl) benzene sulfonamide and 4-amino- $N$-(2,6-dimethoxy pyrimidin-4-yl)benzenesulfonamide) ( $0.01 \mathrm{~mol})$ in dry dioxane (10 $\mathrm{mL}$ ) and a catalytic amount of pyridine was refluxed for $1 \mathrm{~h}$. Then the mixture was left to cool. The solid product that formed was recrystallized from dioxane to give compounds 31a,b (Scheme 6).

$\mathrm{N}$-\{4-[N-(5-Methylisoxazol-3-yl)sulfamoyl]phenyl\}-2-(2-oxo4-phenyl-2H-chromen-7-yloxy)acetamide (31a): Color: White. Yield: $64 \%$. M.p.: $248-250^{\circ} \mathrm{C}$. FT-IR $\left(\mathrm{KBr}, v, \mathrm{~cm}^{-1}\right): 3352,3230$ (NH), $1706(\mathrm{C}=0) .{ }^{1} \mathrm{H}$ NMR (300 MHz, DMSO- $\left.d_{6}, \delta, \mathrm{ppm}\right): 10.56$ (s, 1H, 1NH), $11.31(\mathrm{~s}, 1 \mathrm{H}, 1 \mathrm{NH}), 6.99-7.82(\mathrm{~m}, 12 \mathrm{H}, \mathrm{Ar}-\mathrm{H}), 6.25$ (s, 1H, H-3), 6.11 (s, 1H, CH-oxazole), $4.90\left(\mathrm{~s}, 2 \mathrm{H}, \mathrm{OCH}_{2}\right), 2.28$ (s, $3 \mathrm{H}, \mathrm{CH}_{3}$ ). Anal. calcd. for $\mathrm{C}_{27} \mathrm{H}_{21} \mathrm{~N}_{3} \mathrm{O}_{7} \mathrm{~S}$ : C, 61.01; H, 3.98; N, 7.91. Found: C, 60.90; H, 3.91; N, 7.85\%.

$N$-\{4-[N-(2,6-Dimethoxypyrimidin-4-yl)sulfamoyl]phenyl $\}-2-$ (2-oxo-4-phenyl-2H-chromen-7-yloxy)acetamide (31b): Color: White. Yield: 64\%. M.p.: $240-242^{\circ} \mathrm{C}$. FT-IR (KBr, v, cm$\left.{ }^{-1}\right): 3336$ $(\mathrm{NH}), 1706$ (C=0). ${ }^{1} \mathrm{H}$ NMR (300 MHz, DMSO- $\left.d_{6}, \delta, \mathrm{ppm}\right): 10.56$ (s, $1 \mathrm{H}, 1 \mathrm{NH}$ exchangeable with $\left.\mathrm{D}_{2} \mathrm{O}\right), 11.48(\mathrm{~s}, 1 \mathrm{H}, 1 \mathrm{NH}$ exchangeable with $\left.\mathrm{D}_{2} \mathrm{O}\right), 6.99-7.92(\mathrm{~m}, 12 \mathrm{H}, \mathrm{Ar}-\mathrm{H}), 6.25(\mathrm{~s}, 1 \mathrm{H}$, $\mathrm{H}-3), 5.94\left(\mathrm{~s}, 1 \mathrm{H}, \mathrm{CH}-\right.$ pyrimidine), $4.90\left(\mathrm{~s}, 2 \mathrm{H}, \mathrm{OCH}_{2}\right), 3.86(\mathrm{~s}$, $\left.3 \mathrm{H}, \mathrm{OCH}_{3}\right), 3.75\left(\mathrm{~s}, 3 \mathrm{H}, \mathrm{OCH}_{3}\right)$. Anal. calcd. for $\mathrm{C}_{29} \mathrm{H}_{24} \mathrm{~N}_{4} \mathrm{O}_{8} \mathrm{~S}: \mathrm{C}$, 59.18; H, 4.11; N, 9.52. Found: C, 59.10; H, 4.03; N, 9.44\%.

2.2.22. General procedure for preparation of $N, N^{\prime}-$ bis [2-(2oxo-4-phenyl-2H-chromen-7-yloxy)acetyl]p-phenylene diamine, $N, N^{\prime}$-bis[2-(2-oxo-4-phenyl-2H-chromen-7-yloxy) acetyl] benzidine, $N$, $N^{\prime}$-bis [2-(2-oxo-4-phenyl-2H-chromen-7yloxy) acetyl]ethylenediamine and $N, N^{\prime}$-bis[2-(2-oxo-4phenyl-2H-chromen-7-yloxy)acetyl]4-aminobenzenesulfonamide (32a-d)

A mixture of 2-(2-oxo-4-phenyl-2H-chromen-7-yloxy)acetyl chloride (26) $(0.02 \mathrm{~mol})$ and bifunctional amines namely $(1,4-$ phenylenediamine, benzidine, ethylenediamine and 4aminoben-zenesulfonamide $(0.01 \mathrm{~mol})$ in dry dioxane $(10 \mathrm{~mL})$ and a catalytic amount of pyridine was refluxed for $2 \mathrm{~h}$. The precipitate formed was filtered off hot, washed with ethanol several times, dried and recrystallized from DMF to give compounds 32a-d (Scheme 7).

$N, N^{\prime}$-bis [2-(2-Oxo-4-phenyl-2H-chromen-7-yloxy)acetyl]pphenylene-diamine (32a): Color: Grey. Yield: 83\%. M.p.: 306$308{ }^{\circ} \mathrm{C}$. FT-IR (KBr, v, cm $\left.{ }^{-1}\right): 3434,3348(\mathrm{NH}), 1714(\mathrm{C}=0) .{ }^{1} \mathrm{H}$ NMR (300 MHz, DMSO- $\left.d_{6}, \delta, \mathrm{ppm}\right): 10.13(\mathrm{~s}, 2 \mathrm{H}, 2 \mathrm{NH}$ exchangeable with $\left.\mathrm{D}_{2} \mathrm{O}\right), 7.01-7.58(\mathrm{~m}, 20 \mathrm{H}, \mathrm{Ar}-\mathrm{H}), 6.25(\mathrm{~s}, 2 \mathrm{H}$ $2 \mathrm{H}-3$ ), 4.84 (s, $4 \mathrm{H}, 2 \mathrm{CCH}_{2}$ ). Anal. calcd. for $\mathrm{C}_{40} \mathrm{H}_{28} \mathrm{~N}_{2} \mathrm{O}_{8}$ : C, 72.28; $\mathrm{H}, 4.25 ; \mathrm{N}, 4.21$. Found: $\mathrm{C}, 72.20 ; \mathrm{H}, 4.13 ; \mathrm{N}, 4.12 \%$.

$N, N^{\prime}$-bis [2-(2-Oxo-4-phenyl-2H-chromen-7-yloxy)acetyl] benzidine (32b): Color: Pale yellow. Yield: 85\%. M.p.: 268-270 ${ }^{\circ} \mathrm{C}$. FT-IR $\left(\mathrm{KBr}, v, \mathrm{~cm}^{-1}\right): 3300(\mathrm{NH}), 1714(\mathrm{C}=0) .{ }^{1} \mathrm{H}$ NMR $(300$ MHz, DMSO- $\left.d_{6}, \delta, \mathrm{ppm}\right): 10.28(\mathrm{~s}, 2 \mathrm{H}, 2 \mathrm{NH}), 6.76-7.74(\mathrm{~m}, 24 \mathrm{H}$, $\mathrm{Ar}-\mathrm{H}), 6.26(\mathrm{~s}, 2 \mathrm{H}, 2 \mathrm{H}-3), 4.89\left(\mathrm{~s}, 4 \mathrm{H}, 2 \mathrm{OCH}_{2}\right)$. 


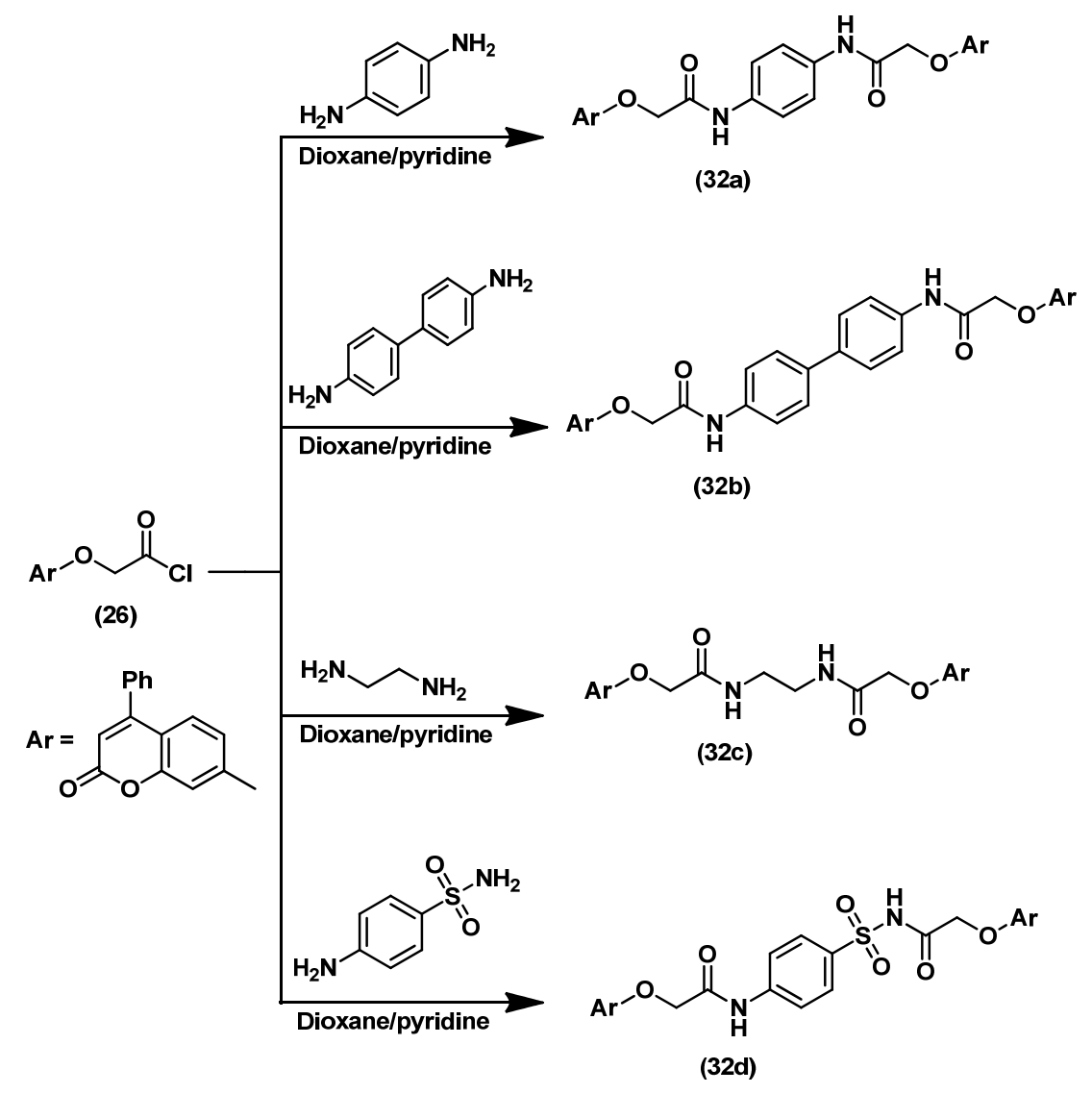

Scheme 7

MS (EI, $m / z(\%)): 740\left(\mathrm{M}^{+}, 56\right)$. Anal. calcd. for $\mathrm{C}_{46} \mathrm{H}_{32} \mathrm{~N}_{2} \mathrm{O}_{8}$ : C, 74.59; H, 4.35; N, 3.78. Found: C, 74.50; H, 4.27; N, 3.67\%. $\mathrm{N}, \mathrm{N}^{\prime}$-bis[2-(2-Oxo-4-phenyl-2H-chromen-7-yloxy)acetyl] ethylenediamine (32c): Color: White. Yield: 80\%. M.p.: 280-282 ${ }^{\circ} \mathrm{C}$. FT-IR (KBr, v, cm-1): $3380(\mathrm{NH}), 1716,1668$ (C=0). ${ }^{1} \mathrm{H}$ NMR $\left(300 \mathrm{MHz}, \mathrm{DMSO}-d_{6}, \delta, \mathrm{ppm}\right): 8.26(\mathrm{~s}, 2 \mathrm{H}, 2 \mathrm{NH}), 6.92-7.56(\mathrm{~m}$, $16 \mathrm{H}, \mathrm{Ar}-\mathrm{H}), 6.27$ (s, 1H, H-3), 6.22 (s, 1H, H-3), 4.66 (s, 2H, $\left.\mathrm{OCH}_{2}\right), 4.60\left(\mathrm{~s}, 2 \mathrm{H}, \mathrm{OCH}_{2}\right), 3.56\left(\mathrm{~s}, 2 \mathrm{H}, \mathrm{CH}_{2}\right), 3.39\left(\mathrm{~s}, 2 \mathrm{H}, \mathrm{CH}_{2}\right)$. MS (EI, $m / z(\%)): 616\left(\mathrm{M}^{+}, 8\right)$. Anal. calcd. for $\mathrm{C}_{36} \mathrm{H}_{28} \mathrm{~N}_{2} \mathrm{O}_{8}$ : C, 70.12; H, 4.58; N, 4.54. Found: C, 70.06; H, 4.50; N, 4.47\%.

$\mathrm{N}, \mathrm{N}^{\prime}$-bis[2-(2-Oxo-4-phenyl-2H-chromen-7-yloxy)acetyl]4aminobenzene-sulfonamide (32d): Color: White. Yield: 73\%. M.p.: 252-254 ${ }^{\circ}$ C. FT-IR (KBr, v, cm-1): 3392, $3336(\mathrm{NH})$ 1698(C=0). ${ }^{1} \mathrm{H}$ NMR (300 MHz, DMSO- $\left.d_{6}, \delta, \mathrm{ppm}\right): 10.48(\mathrm{~s}, 2 \mathrm{H}$, 2NH), 7.04-7.79 (m, 20H, Ar-H), 6.26 (s, 2H, 2H-3), 4.91 (s, 4H, $\left.2 \mathrm{CH}_{2}\right)$. MS (EI, $\left.m / z(\%)\right): 728\left(\mathrm{M}^{+}, 27\right)$. Anal. calcd. for $\mathrm{C}_{40} \mathrm{H}_{28} \mathrm{~N}_{2} \mathrm{O}_{10} \mathrm{~S}: \mathrm{C}, 65.93 ; \mathrm{H}, 3.87 ; \mathrm{N}, 3.84$. Found: $\mathrm{C}, 65.87 ; \mathrm{H}, 3.80$; N, $3.77 \%$.

\subsection{Pharmacology}

\subsubsection{2,2-Diphenyl-1-picrylhydrazyl (DPPH) radical scavenging activity}

In the DPPH assay, antioxidants reduce the free radical 2,2diphenyl-1- picrylhydrazyl. In the presence of an antioxidant, the purple color of $\mathrm{DPPH} \cdot$ fades and the change of absorbance can be followed spectrophotometrically at $515 \mathrm{~nm}$ [27].

Test compounds are dissolved in $\mathrm{N}, \mathrm{N}$-dimethylformamide (DMF) solution to specific concentrations, and each sample was mixed with DPPH in DMF solution, the blank sample contains the same amount of DMF and DPPH. The mixtures were left for
$15 \mathrm{~min}$ at $25^{\circ} \mathrm{C}$ then the absorbance measured at $517 \mathrm{~nm}$ using the UV-vis spectrophotometer. Generally, the results are reported as the percent of inhibition, i.e. the amount of antioxidant necessary to decrease the initial $\mathrm{DPPH}^{-}$ concentration by $50 \%$. The percentage of DPPH radical scavenger was calculated using the equation (1), [28].

The percentage of DPPH radical scavenger $=\left[\left(\mathrm{A}_{0}-\mathrm{A}_{\mathrm{t}}\right) / \mathrm{A}_{0}\right] \times 100$

Where, $A_{0}$ is the absorbance value of blank sample, at a particular time and $A_{t}$ is the absorbance value of the tested sample. The $\mathrm{IC}_{50}$ (concentration causing $50 \%$ inhibition of $\mathrm{DPPH}$ ) values of each test compound are determined. Ascorbic acid can be used as positive controls.

\subsubsection{Cytotoxicity assay in-vitro}

The cytotoxicity of the drugs will be tested against human liver cancer cell line (HepG2) by SRB assay as described by Sumangala et al. [29]. Exponentially growing cells will be collected using $0.25 \%$ Trypsin-EDTA and plated in 96-well plates at 1000-2000 cells/well. Cells will be exposed to the drugs for $72 \mathrm{~h}$ and subsequently fixed with TCA (10\%) for $1 \mathrm{~h}$ at $4{ }^{\circ} \mathrm{C}$. After several washings, cells will be exposed to $0.4 \%$ SRB solution for $10 \mathrm{~min}$ in dark place and subsequently washed with $1 \%$ glacial acetic acid. After drying overnight, Tris- $\mathrm{HCl}$ will be used to dissolve the SRB-stained cells and color intensity will be measured at 492 and $630 \mathrm{~nm}$ (for the reference wavelength) with the enzyme linked immune sorbent assay (ELISA) reader. All of the compounds were tested in twice in each of the cell lines. 


\section{Results and discussion}

\subsection{Synthesis}

Hydrazinolysis of ethyl 2-(2-oxo-4-phenyl-2H-chromen-7yloxy)acetate (1)with hydrazine hydrate in ethanol at refluxing temperature,2-(2-oxo-4-phenyl-2 $\mathrm{H}$-chromen-7-yloxy)aceto hydrazide (2)was obtained in good yield (Scheme 1).

Interaction of acetohydrazide2 with carbon disulfide in refluxing ethanol in the presence of potassium hydroxide resulted in the formation of the potassium salt of hydrazinecarbodithioate, 3 . Which on treatment with aqueous potassium hydroxide solution under reflux then neutralized with hydrochloric acid afforded a product identified as 7-[(5thioxo-1,3,4-oxadiazol-2-yl)-methoxy]-4-phenyl- $2 H$-chromen-

2-one (4). Treatment of the potassium salt $\mathbf{3}$ or $\mathbf{4}$ with hydrazine hydrate in aqueous ethanol yielded the corresponding 7-[(4-amino-5-thioxo-4,5-dihydro- $1 \mathrm{H}-1,2,4-$ triazol-3-yl)methoxy]-4-phenyl-2H-chromen-2-one (5).

Interaction of the potassium salt $\mathbf{3}$ with 4-nitrophenacyl bromide in refluxing DMF furnished the expected product that identified as $\mathrm{N}$-[4-(4-nitrophenyl)-2-thioxothiazol-3 $(2 \mathrm{H})$-yl)-2(2-oxo-4-phenyl-2H-chromen-7-yloxy] acetamide (7),the formation of this compound was shown through the proposed mechanism outline in Scheme 1. Treatment of compound4with 4-nitrophenacyl bromide in refluxing acetone in the presence of anhydrous potassium carbonate afforded a single product which identified as 7-\{[5-(2-(4-nitrophenyl)-2-oxoethylthio)1,3,4-oxadiazol-2-yl]methoxy\}-4-phenyl- $2 \mathrm{H}$-chromen-2-one (8). Compound $\mathbf{1 0}$ was obtained by treatment of compound $\mathbf{5}$ with $p$-nitrophenacyl bromide in refluxing DMF in the presence of triethylamine. The formation of compound $\mathbf{1 0}$ was explained by the nucleophilic transformation into acyclic non-isolable intermediate (9) that undergoes intramolecular ring closure to the final product through nucleophilic addition of amino $\left(\mathrm{NH}_{2}\right)$ to the carbonyl group followed by elimination of water molecule to furnish the expected product7-\{[6-(4-nitrophenyl)$7 H$-[1,2,4] triazolo[3,4-b][1,3,4] thiadia-zin-3-yl]methoxy\}-4phenyl- $H$-chromen-2-one (10). The structure of this compound was further confirmed by its alternative synthesis from the reaction of the 1,3,4-oxadiazole derivative 8 with hydrazine hydrate in refluxing ethanol afforded a product which identical in all aspects (M.p., mixed m.p. and spectral data) with compound 10, (Scheme 1).

Condensation of 7-[(4-amino-5-thioxo-4,5-dihydro- $1 H-1,2$, 4-triazol-3-yl)methoxy]-4-phenyl-2H-chromen-2-one(5)with 4nitrobenzaldehyde in refluxing ethanol containing a few drops from acetic acid gave the corresponding imine 11, rather than the expected product triazolothiadiazolyl derivative12, (Scheme 2).

Also, the acetohydrazide 2 was reacted with some anhydrides such as phthalic anhydride and pyrazine-2,3dicarboxylicanhydride in refluxing glacial acetic acid to give the corresponding imides which formulated as $\mathrm{N}$-(substituted)-2(2-oxo-4-phenyl-2H-chromen-7-yloxy)acetamide $\quad(\mathbf{1 3 a}, \mathbf{b})$, respectively. Treatment of compound $\mathbf{2}$ with phenylisothio cyanate and phenylisocyanate in refluxing dioxane gave 4phenyl-7-[(4-phenyl-5-thioxo-4,5-dihydro- $1 H$-1,2,4-triazol-3yl)methoxy]-2 $H$-chromen-2-one (15a) and 3-[(2-oxo-4-phenyl$2 H$-chromen-7-yloxy)methyl]-4-phenyl-1 $H$-1,2,4-triazol-5(4H)one $(\mathbf{1 5 b})$, respectively. The formation of these compounds were assumed to proceed via nucleophilic addition of amino group in compound $\mathbf{2}$ to isothiocyanate and/or isocyanate to give acyclic non-isolable semicarbazide intermediate 14a,b which underwent intramolecular cyclization by elimination of water molecule to furnish the final products 15a,b, respectively. Acetohydrazide 2 reacted with 4-amino-2mercapto-6-(4-methoxyphenyl) pyrimidine-5-carbonitrile (16) [30] in refluxing DMF in the presence of a few drops from pyridine to give $N^{\prime}$-[4-amino-5-cyano-6-(4-methoxyphenyl)
pyrimidin-2-yl]-2-(2-oxo-4-phenyl-2H-chromen-7-yloxy)aceto hydrazide (17), (Scheme 3).

The reaction of hydrazide2 with 4-benzyl-1-chloro phthalazine (18)[31] in boiling dioxane in the presence of pyridine furnished 7-[(6-benzyl-[1,2,4]triazolo[4,5-a] phthalazin-3-yl)methoxy]-4-phenyl-2H-chromen-2-one (20) as the only isolable product. The formation of this compound was explained by the nucleophilic transformation into acyclic nonisolable intermediate 19 which undergoes intramolecular ring closure to the final product via elimination of water molecule, while treatment of hydrazide 2 with chloroacetonitrile in dry dioxane-pyridine under reflux yielded a product which identified as 7-[(6-amino-4H-1,3,4-oxadiazin-2-yl)methoxy]-4phenyl-2H-chromen-2-one (22). The formation of this compound was assumed by nucleophilic substitution to form acyclic non-isolable intermediate $\mathbf{2 1}$ followed by intramolecular attack at the cyanide group to form the cyclized product 22 (Scheme 4).

On the other hand, when ethyl 2-(2-oxo-4-phenyl-2H-chromen-7-yloxy)acetate (1) was treated with sodium hydroxide in refluxing ethanol yielded the corresponding acid which identified as 2-(2-oxo-4-phenyl- $2 H$-chromen-7-yloxy)acetic acid (23) [26].

7-((4,5-Dihydro-1H-imidazol-2-yl)methoxy)-4-phenyl- $2 \mathrm{H}$ chromen-2-one (24) was prepared by fusion of ethylene diamine with compound 23, while cyclocondensation of compound 23 with (1,2-phenylenediamine, 2-aminophenol and 2-aminothiophenol) under fusion conditions yielded products which identified as 7-[(substituted)methoxy]-4-phenyl-2Hchromen-2-one (25a-c). Treatment of compound 23 with thionyl chloride gave the corresponding acid chloride as $2-(2-$ oxo-4-phenyl-2H-chromen-7-yloxy) acetyl chloride (26) (Scheme 5).

The acid chloride $\mathbf{2 6}$ was allowed to react with different aromatic amines in dry dioxane containing a few drops of pyridine under reflux afforded products which identified as: $\mathrm{N}$ (4-acetylphenyl)-2-(2-oxo-4-phenyl- $2 H$-chromen-7-yloxy) acetamide (27a), 2-\{4-[2-(2-oxo-4-phenyl- $2 H$-chromen-7yloxy)acetamido]-phenyl\}acetic acid (27b) and 4-[2-(2-oxo-4phenyl-2H-chromen-7-yloxy)acetamido]benzoic acid (27c), respectively. Furthermore, whencompound $\mathbf{2 6}$ was subjected to the reaction with 4-amino benzenesulfonic acid in refluxing dioxane-pyridine yielded asingle product which identified as: 4-[2-(2-oxo-4-phenyl- $2 \mathrm{H}$-chromen-7-yloxy)acetamido] benzenesulfonic acid (28), while compound $\mathbf{2 6}$ reacted with anthranilic acid and methyl anthranilate in boiling dioxanepyridineto give the products which were found to be $2-[2-(2-$ oxo-4-phenyl- $2 \mathrm{H}$-chromen-7-yloxy)acetamido] benzoic acid (29a) and methyl 2-[2-(2-oxo-4-phenyl-2H-chromen-7-yloxy) acetamido]benzoate $(\mathbf{2 9 b})$, respectively. Also, reaction of acid chloride 26 with 2-(2-oxo-4-phenyl- $2 H$-chromen-7-yloxy) acetohydrazide (2) in dry dioxane containing a few drops from pyridine at reflux temperature afforded bis acyl hydrazine derivative $\mathbf{3 0}$. The acid chloride $\mathbf{2 6}$ was reacted with sulfa drug derivatives under the same reaction conditions to produce $N$ \{4-[N-(substituted)sulfamoyl] phenyl\}-2-(2-oxo-4-phenyl-2Hchromen-7-yloxy)acetamide (31a,b) (Scheme 6).

Finally, this investigation was extended to include the reactivity of 2-(2-oxo-4-phenyl- $2 H$-chromen-7-yloxy)acetyl chloride (26) towards bifunctional isolated amines to furnish the corresponding bis compounds (32a-d) (Scheme 7).

\subsection{Pharmacology}

\subsubsection{Antioxidant activity}

Antioxidant compounds in food play an important role as a health-protecting factor. Scientific evidence suggests that antioxidants reduce the risk for chronic diseases. The role of antioxidant is to remove free radicals, by donating hydrogen to free radicals in its reduction to produce non-reactive species. 
Many synthetic antioxidant compounds have shown toxic and/or mutagenic effects; therefore attention has been paid to naturally occurring antioxidants. Test compounds were screened for in vitro antioxidant activity using DPPH.

The effect of the different synthetic compounds on DPPH radical scavenging was compared to ascorbic acid using as positive control and appreciated by the determination of the $\mathrm{IC}_{50}$ values. The results are listed in Table 1.

DPPH test is a direct and reliable method for determining radical scavenging action. The DPPH radical contains an odd electron, which is responsible for the absorbance at 515-517 $\mathrm{nm}$ and also for a visible deep purple color. When DPPH accepts an electron donated by an antioxidant compound, the DPPH is decolorized, which can be quantitatively measured from the changes in absorbance.

The ratio of antioxidant/DPPH required to decrease the concentration of DPPH to $50 \%$ of its initial value, denoted as $\mathrm{IC}_{50}$ or Efficient Concentration (EC $\mathrm{E}_{50}$ ), is an indicator of antiradical activity [32]. It is clear from the tabulated results that compound 10showed the best antioxidant activity against DPPH intested compounds, while compounds 29b, 31a and 32a showed no activity. In concentration $1000 \mu \mathrm{g} / \mathrm{mL}$, we noted that derivative $\mathbf{1 0}$ has most potent scavenging behavior among test compounds and also more potent than ascorbic acid. Similarly, $\mathbf{2 8}$ and $\mathbf{3 2 b}$ have scavenging activity more than ascorbic acid (Figure 1). However, compound $\mathbf{4}$ have scavenging activity similar to ascorbic acid.

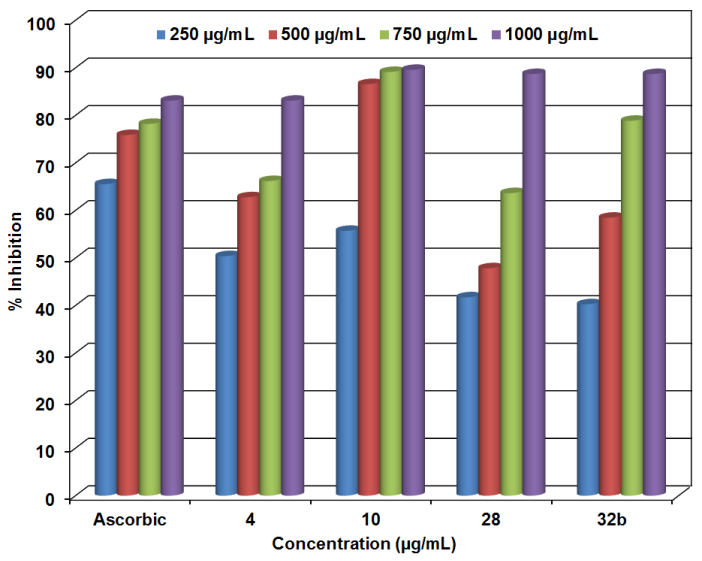

Figure 1. Effect of compounds 4, 10, 28 and 32b toward 2,2-diphenyl-1picrylhydrazyl (DPPH).

\subsubsection{Cytotoxicity assay in vitro}

The results expressed as $\mathrm{IC}_{50}$ (inhibitory concentration of $50 \%$ ) were the averages of two determinations and were calculated by using igmoidal concentration-response curve fitting models (Sigma Plot software). The results are listed in Table 2 .

This study revealed that 7-\{[6-(4-nitrophenyl)-7 $H$ $[1,2,4]$ tria-zolo $[3,4-b][1,3,4]$ thiadiazin-3-yl]methoxy $\}-4$ phenyl- $H$-chromen-2-one (10) derivative is the most potent $\left(\mathrm{IC}_{50}(\mu \mathrm{g} / \mathrm{mL})=9.067\right)$, while derivatives 28 and $\mathbf{3 2 b}$ are similar $\left(\mathrm{IC}_{50}(\mu \mathrm{g} / \mathrm{mL})=18.56\right)$.

\section{Conclusions}

Our interest in synthesis of heterocyclic compounds is to focus on their biological activity as a part of our program, which aimed at the development of new and more potent antioxidant and anticancer agents. Thus, in this paper, we revealed the synthesis of some coumarin derivatives and biological evaluation of some novel compounds.
Table 1. Values of IC $\mathrm{I}_{50}$ exhibited by test compounds.

\begin{tabular}{|c|c|c|c|c|}
\hline Entry & $\begin{array}{l}\text { Compound } \\
\text { no }\end{array}$ & $\begin{array}{l}\text { Concentration } \\
(\mu \mathrm{g} / \mathrm{mL})\end{array}$ & $\begin{array}{l}\% \\
\text { Inhibition }\end{array}$ & $\begin{array}{l}\mathrm{IC}_{50} \\
(\mu \mathrm{g} / \mathrm{mL})\end{array}$ \\
\hline \multirow[t]{4}{*}{1} & 4 & 250 & 50.40 & 263 \\
\hline & & 500 & 62.78 & \\
\hline & & 750 & 66.21 & \\
\hline & & 1000 & 83.10 & \\
\hline \multirow[t]{4}{*}{2} & 7 & 250 & 31.17 & 6118 \\
\hline & & 500 & 34.16 & \\
\hline & & 750 & 36.56 & \\
\hline & & 1000 & 39.33 & \\
\hline \multirow[t]{4}{*}{$\overline{3}$} & 10 & 250 & 55.72 & 213 \\
\hline & & 500 & 86.60 & \\
\hline & & 750 & 89.15 & \\
\hline & & 1000 & 89.58 & \\
\hline \multirow[t]{4}{*}{4} & $13 \mathrm{~b}$ & 250 & 19.83 & 1473 \\
\hline & & 500 & 24.61 & \\
\hline & & 750 & 35.30 & \\
\hline & & 1000 & 43.18 & \\
\hline \multirow[t]{4}{*}{5} & $15 b$ & 250 & 26.02 & 1433 \\
\hline & & 500 & 36.99 & \\
\hline & & 750 & 42.19 & \\
\hline & & 1000 & 43.32 & \\
\hline \multirow[t]{4}{*}{6} & 24 & 250 & 45.88 & 123 \\
\hline & & 500 & 38.60 & \\
\hline & & 750 & 37.80 & \\
\hline & & 1000 & 36.56 & \\
\hline \multirow[t]{4}{*}{7} & $25 a$ & 250 & 37.44 & 114 \\
\hline & & 500 & 34.89 & \\
\hline & & 750 & 28.84 & \\
\hline & & 1000 & 19.30 & \\
\hline \multirow[t]{4}{*}{8} & $25 c$ & 250 & 52.66 & 317 \\
\hline & & 500 & 44.65 & \\
\hline & & 750 & 38.02 & \\
\hline & & 1000 & 31.54 & \\
\hline \multirow[t]{4}{*}{9} & $27 \mathrm{~b}$ & 250 & 37.36 & 5380 \\
\hline & & 500 & 39.40 & \\
\hline & & 750 & 39.62 & \\
\hline & & 1000 & 44.21 & \\
\hline \multirow[t]{4}{*}{10} & $27 c$ & 250 & 39.40 & 683792 \\
\hline & & 500 & 39.55 & \\
\hline & & 750 & 40.13 & \\
\hline & & 1000 & 41.51 & \\
\hline$\overline{11}$ & 28 & 250 & 41.73 & 397 \\
\hline & & 500 & 47.85 & \\
\hline & & 750 & 63.66 & \\
\hline & & 1000 & 88.71 & \\
\hline 12 & $29 b$ & 250 & 39.04 & Inactive \\
\hline & & 500 & 38.82 & \\
\hline & & 750 & 38.09 & \\
\hline & & 1000 & 37.73 & \\
\hline 13 & $31 a$ & 250 & 40.79 & Inactive \\
\hline & & 500 & 40.93 & \\
\hline & & 750 & 41.88 & \\
\hline & & 1000 & 42.61 & \\
\hline 14 & $31 b$ & 250 & 39.91 & 11663 \\
\hline & & 500 & 40.57 & \\
\hline & & 750 & 41.81 & \\
\hline & & 1000 & 43.99 & \\
\hline 15 & $32 a$ & 250 & 39.48 & Inactive \\
\hline & & 500 & 39.11 & \\
\hline & & 750 & 37.58 & \\
\hline & & 1000 & 36.13 & \\
\hline$\overline{16}$ & $32 b$ & 250 & 40.28 & 344 \\
\hline & & 500 & 58.49 & \\
\hline & & 750 & 78.88 & \\
\hline & & 1000 & 88.71 & \\
\hline 17 & $32 d$ & 250 & 32.77 & 2590 \\
\hline & & 500 & 36.57 & \\
\hline & & 750 & 41.35 & \\
\hline & & 1000 & 42.33 & \\
\hline Std. & Ascorbic & 250 & 65.55 & 91.45 \\
\hline & acid & 500 & 75.89 & \\
\hline & & 750 & 78.22 & \\
\hline & & 1000 & 83.10 & \\
\hline
\end{tabular}

The structures of the newly compounds were elucidated on the basis of IR, ${ }^{1} \mathrm{H}$ NMR, ${ }^{13} \mathrm{C}$ NMR and MS spectral data. A series of novel coumarin derivatives were prepared. The antioxidant 
and anticancer activity of some compounds were evaluated. 7\{[6-(4-Nitrophenyl)-7H-[1,2,4] tria-zolo[3,4-b][1,3,4]thiadiazin3-yl] methoxy $\}$-4-phenyl- $H$-chromen-2-one $(\mathbf{1 0})$ has the highest antioxidant and anticancer activity in screened compounds.

\begin{tabular}{lll}
\multicolumn{3}{l}{ Table 2. Cytotoxicity assay in vitro for some synthesized compounds. } \\
\hline Entry & Compounds & IC $_{50}(\boldsymbol{\mu g} / \mathbf{m L})$ \\
\hline 1 & 4 & 14.48 \\
2 & 10 & 9.067 \\
3 & 28 & 18.56 \\
4 & $32 \mathrm{~b}$ & 18.56 \\
\hline
\end{tabular}

\section{Acknowledgements}

This study was supported by the Chemistry Department, Faculty of Science, Al-Azhar University. We are deeply thankful to Micro Analytical Center for making the IR, ${ }^{1} \mathrm{H}$ NMR, ${ }^{13} \mathrm{C}$ NMR and MS samples.

\section{References}

[1]. Chakraborty, D. P.; Chatterji, D. J. Org. Chem. 1969, 34(12), 3784-3786.

[2]. Finnegan, R. A.; Morris, M. P.; Djerassi, C. J. Org. Chem. 1961, 26(4), 1180-1184.

[3]. Al-Amiery, A. A.; Ahmed, Y. M.; Abdul Amir, H. K.; Abu Bakar, M. Molecules 2011, 16, 6833-6843.

[4]. Holho, D.; Boschetti, E.; Lipha, Fr. Pat. No. 1, 310, 535; 1963.

[5]. Meguro, K.; Tawada, H.; Jpn. Pat. No. 90129, 240; 1991.

[6]. Anderson, D. M.; Shelley, S.; Crick, N.; Buraglio, M. J. Clin. Pharmacol. 2002, 42, 1358-1365.

[7]. Tassies, D.; Freire, C.; Puoan, J.; Maragall, S.; Monteagudo, J. Haematologica 2002, 87, 1185-1191.

[8]. Vittorio, F.; Ronsisvalle, G.; Marrazzo, A.; Blandini, G. Farmaco 1995, 50, 265-272.

[9]. Nayyar, V.; Monga, A.; Malde, E.; Coutinho, R. Bioorg. Med. Chem. 2007, 15, 626-640.

[10]. Sztanke, K.; Pasterhak, K.; Rzymowska, J.; Sztanke, M.; KandeferSzerszen, M. Eur. J. Med. Chem. 2007, 43, 404-419.

[11]. Radhwan, M. A. A.; Ragab, E. A.; Sabry, N. M.; El-Shenawy, S. M. Bioorg. Med. Chem. 2007, 15, 3832-3841.

[12]. Gemma, S.; Kukreja, G.; Fattorusso, C.; Persico, M.; Romano, M. P.; Altarelli, M.; Savini, L.; Campiani, G.; Fattorusso, E.; Basilico, N.; Taramelli, D.; Yardley, V.; Butini, S. Bioorg. Med. Chem. Lett. 2006, 16, 5384-5388.

[13]. Al-Mawsawi, L. Q.; Dayam, R.; Taheri, L.; Witvrouw, M.; Debyser, Z.; Neamati, N. Bioorg. Med. Chem. Lett. 2007, 17, 6472-6475.

[14]. Hansch, C.; Fujita, T. J. Am. Chem. Soc. 1964, 86, 1616-1626.

[15]. Kamal, M. D.; Ahmad, M. F.; Hatem, A. A. Heteroatom. Chem. 2005, 16, 621-627.

[16]. Abdel-Al, E. H.; Al-Ashamawi, M. I.; Abd El-Fattah, B. Arch. Pharm. 1983, 38, 833-838.

[17]. Gupta, A.K.S.; Garg, M.; Chandra, U. J. Indian Chem. Soc. 1979, 56, 1230 1232.

[18]. Mansour, A. K.; Eid, M. M.; Khalil, N. S. A. M. Molecules 2003, 8, 744755.

[19]. Walser, A.; Flynn, T.; Mason, C. J. Heterocycl. Chem. 1991, 28, 11211125.

[20]. Reiter, J.; Pongo, L.; Kovesdi, I.; Pallagi, I. J. Heterocycl. Chem. 1995, 32, 407-417.

[21]. Anderson, D. K.; Deuwer, D. L.; Sirkorski, J. A. J. Heterocycl. Chem. 1995, 32, 893-898.

[22]. Collin, X.; Sauleau, A.; Coulon, J. Bioorg. Med. Chem. Lett. 2003, 13, 2601-2605.

[23]. Goswami, B. N.; Kataky, J. C. S.; Baruah, J. N. J. Indian Chem. Soc. 1987, $64,422-424$.

[24]. Hirota, T.; Sasaki, K.; Yamamoto, H.; Nakayama, T. J. Heterocycl. Chem. 1991, 28, 257-261.

[25]. Al-Soud, Y. A.; Al-Masoudi, N. A.; Ferwanah, A. E. S. Bioorg. Med. Chem. 2003, 11, 1701-1708.

[26]. Garazd, M. M.; Garazd, Ya. L.; Smirnov, M. N.; Khilya, V. P. Chem. Nat. Compd. 1999, 35, 415-419.

[27]. Schlesier, K.; Harwat, M.; Böhm, V.; Bitsch, R. Free Radic. Res. 2002, 36, 177-187.

[28]. Kadhum, A. H.; Al-Amiery, A. A.; Musa, A. Y.; Mohamad, A. B. Int. J. Mol. Sci.2011, 12, 5747-5761.

[29]. Sumangala, V.; Boja, P.; Chidananda, N.; Arulmoli, T.; Shalini, S. Eur. J. Med. Chem. 2012, 54, 59-64.

[30]. El-Agrody, A. M.; Ali, F. M.; Eid, F. A.; El-Nassag, M. A.; El-Sherbeny, A. G.; Bedair, A. H. Phosphorous Sulfur 2006, 18, 839-864.
[31]. Abd El-Wahab, A. H. F.; Mohamed, H. M.; El-Agrody, A. M.; El-Nassag, M. A.; Bedair, A. H. Eur. J. Chem. 2013, 4, 10-19.

[32]. Brand-Williams, W.; Cuvelier, M. E.; Berset, C. Lebensm. Wiss. Technol. 1995, 28, 25-30. 